\title{
LOS RENDIMIENTOS \\ DE LOS SISTEMAS ELECTORALES DE LAS COMUNIDADES AUTÓNOMAS: EL PREDOMINIO DEL BIPARTIDISMO IMPERFECTO*
}

\author{
Francisco J. Llera Ramo \\ Universidad del País Vasco
}

\begin{abstract}
RESUMEN
Los rasgos básicos del sistema electoral español han cumplido ya veinte años y su generalización a nivel regional alrededor de quince, lo que permite afirmar su institucionalización. En un Estado descentralizado políticamente, el estudio del rendimiento del sistema electoral tiene que referirse necesariamente a su influencia genérica sobre el funcionamiento del sistema político y, más en concreto, sobre el comportamiento electoral, la representación, el sistema de partidos y la gobernabilidad. Se trata, por tanto, de contribuir al esclarecimiento de los términos del debate sobre el sistema electoral español, sobre la base de un mejor conocimiento de los efectos electorales y las consecuencias políticas de los sistemas electorales de las 17 Comunidades Autónomas. Más en concreto, se abordan los siguientes aspectos: los rasgos básicos y diferenciales de los sistemas electorales regionales, las tallas y coeficientes de representación de los respectivos parlamentos, la desigualdad y desproporcionalidad del voto en relación a la magnitud de los distritos, las barreras mínimas de voto y los umbrales de inclusión, la comparación de los principales indicadores de desproporcionalidad y, finalmente, los formatos de los distintos sistemas de partidos regionales y los modelos de gobernabilidad resultantes.
\end{abstract}

Los rasgos básicos del sistema electoral español han cumplido ya veinte años y su generalización a nivel autonómico alrededor de quince años, lo que

* Una primera versión de este trabajo fue presentada como ponencia en las Jornadas del Parlamento de Andalucía sobre «El sistema electoral a debate», celebradas en Sevilla (27-29 de noviembre de 1996). 
permite afirmar su institucionalización. El estudio del rendimiento de un sistema electoral tiene que referirse necesariamente a su influencia genérica sobre el funcionamiento del sistema político. Tal incidencia se relaciona con su principal función de producir representación a través de la conversión de votos en escaños, con efectos, por un lado, reguladores de la competencia electoral, tanto desde el punto de vista de la psicología de los electores como desde la orientación de las estrategias de las élites políticas, pero, por otro lado, conformadores, más o menos mecánicamente, del sistema de partidos, que tiene que producir gobernabilidad. En el caso de una democracia que comienza o, más en concreto, en el caso de la institucionalización de su formato autonómico de descentralización, cabe atribuir al sistema electoral una importancia básica en su desarrollo institucional.

La Constitución de 1978 ha implantado un modelo descentralizado de la forma de Estado, que hemos llamado Estado de las Autonomías y que ha supuesto la creación de 17 Parlamentos regionales con sus respectivos gobiernos desde 1980. La regulación de la competencia política y la producción de representación en tal número de arenas políticas han generado la coexistencia de distintos sistemas electorales en cada una de las Comunidades Autónomas, produciendo incluso distintos sistemas de partidos en algunas de ellas, por la relevancia adquirida por el cleavage nacionalista o regionalista, y dando lugar a lo que se ha denominado Españas electorales (Vallès, 1987 y 1991) para reflejar la heterogeneidad de los nuevos alineamientos electorales y partidistas sobre distintas bases territoriales.

En los apartados que siguen trataré de contribuir al esclarecimiento de los términos del debate sobre el sistema electoral español, sobre la base de un mejor conocimiento de los efectos electorales y las consecuencias políticas de los sistemas electorales de las Comunidades Autónomas. En primer lugar, una visión panorámica de la normativa vigente permitirá caracterizar los rasgos básicos y diferenciales de cada uno de los 17 sistemas electorales. En el segundo apartado se compararán las tallas y los coeficientes de representación de los Parlamentos autónomos. En tercer lugar, se evaluará la desigualdad y desproporcionalidad del voto en relación a los sistemas de distritos, que se ha mostrado como uno de los factores más críticos del sistema electoral español en su conjunto. El cuarto epígrafe evaluará la aplicación de las barreras mínimas de voto y los umbrales de inclusión resultantes. En el quinto apartado se hará un estudio comparado de los principales indicadores de la desproporcionalidad entre votos y escaños. $\mathrm{Y}$, finalmente, el sexto y último estará dedicado a la caracterización de los formatos de los distintos sistemas de partidos y los modelos de gobernabilidad resultantes. 


\section{LOS SISTEMAS ELECTORALES EN LOS ESTATUTOS Y EN LAS LEYES ELECTORALES DE LAS COMUNIDADES AUTÓNOMAS}

Las Comunidades Autónomas regulan, de una u otra forma, el sistema electoral mediante el cual designan los componentes de sus respectivas asambleas legislativas. Aunque se han señalado las ambigüedades y las reservas constitucionales sobre la competencia en materia de regulación del proceso electoral (Fernández, 1981), lo cierto es que todas las Comunidades han legislado en esta materia. Los criterios han oscilado entre el afán mimético respecto del sistema implantado para el Congreso de los Diputados y el deseo de generalizar un sistema relativamente homogéneo tras los pactos autonómicos entre UCD y PSOE (Vallès, 1988; Groppi, 1991). Tales tendencias cristalizaron con tanta intensidad en los textos jurídicos autonómicos que una reforma urgente de la LOREG en marzo de 1991, homogeneizando la convocatoria de elecciones regionales, obligó a la modificación simultánea de los Estatutos de Autonomía de Asturias, Cantabria, Castilla-La Mancha, Comunidad Valenciana, Extremadura, Madrid y Murcia.

La mayor parte de las Comunidades Autónomas, siguiendo la técnica legislativa iniciada por la Constitución, incluyen en el articulado de sus respectivos Estatutos de Autonomía, como se puede comprobar en la tabla 1, una parte importante de los elementos del sistema electoral, haciéndolos por tanto más resistentes a ulteriores reformas. En esta tabla hemos recogido sólo las referencias que aparecen en el articulado, excluyendo del análisis las disposiciones transitorias. La primera diferencia básica estriba en la convocatoria de elecciones y en la duración del mandato parlamentario, con o sin posibilidad de disolución anticipada. Así, Andalucía, Cataluña, Galicia y el País Vasco se diferencian de todas las demás, no sólo por tener su propio ritmo parlamentario, sino también por disponer de la potestad presidencial para disolver sus Parlamentos y anticipar las elecciones. Por el contrario, las restantes Comunidades Autónomas $^{1}$ de vía lenta se han visto obligadas a sincronizar y homogeneizar sus ritmos de celebración y convocatoria electorales, haciéndolas coincidir cada cuatro años y desde 1983 con las elecciones locales del último domingo del mes de mayo, independientemente de que se puedan producir crisis y cambios de mayoría a lo largo de la legislatura (Revenga, 1988).

Esta primera diferencia, junto con la mayor especificidad de sus sistemas de partidos, sobre todo en Cataluña y el País Vasco, dotan de mayor relevancia política a las elecciones autonómicas de las primeras respecto de las de régimen

En los días en que se cierra la redacción de este análisis se está iniciando la reforma de algunos Estatutos de Autonomía, habiendo sido aprobadas ya por el Senado (4 de diciembre de 1996) las reformas de los de Canarias y Aragón, que, además de reconocerles el calificativo de «nacionalidad», confieren al presidente del ejecutivo autónomo la capacidad para disolver su parlamento y convocar nuevas elecciones, lo que puede servir de referencia para la reforma de los otros Estatutos del art. 143. 
TABLA 1

Elementos del sistema electoral contenidos en los Estatutos de Autonomía

\begin{tabular}{|c|c|c|c|c|c|c|c|c|}
\hline Comunidad & $\begin{array}{l}\text { Referencia } \\
\text { legislativa }\end{array}$ & $\begin{array}{l}\text { Tamaño } \\
\text { Parlamento }\end{array}$ & $\begin{array}{l}\text { Fórmula } \\
\text { escrutinio }\end{array}$ & $\begin{array}{l}\text { Delimitación } \\
\text { distritos }\end{array}$ & $\begin{array}{l}\text { Magnitud } \\
\text { distritos }\end{array}$ & $\begin{array}{l}\text { Barreras } \\
\text { minimas }\end{array}$ & $\begin{array}{l}\text { Denominación } \\
\text { Parlamento }\end{array}$ & $\begin{array}{l}\text { Disolución } \\
\text { anticipada }\end{array}$ \\
\hline Andalucía & $\begin{array}{l}\text { L.O. 6/1981, } 30 \text { dic. } \\
\text { (BOJA 12, 6-2-82) }\end{array}$ & $\begin{array}{l}90 / 110 \\
\text { art. } 26.1\end{array}$ & $\begin{array}{l}\text { Id. Congr. Dip. } \\
\text { art. } 28.2\end{array}$ & $\begin{array}{l}\text { Provinciales } \\
\text { art. } 28.2\end{array}$ & $\begin{array}{l}\text { Máximo } \\
\text { art. } 28.1\end{array}$ & - & $\begin{array}{l}\text { Parlamento de } \\
\text { Andalucía }\end{array}$ & Sí \\
\hline Aragón & $\begin{array}{l}\text { L.O. } 8 / 1982,10 \text { agos. } \\
\text { (BOE } 195,16-8-82)\end{array}$ & $\begin{array}{l}60 / 75 \\
\text { art. } 19\end{array}$ & $\begin{array}{l}\text { Proporcional } \\
\text { art. } 18.3\end{array}$ & $\begin{array}{l}\text { Provinciales } \\
\text { art. } 18.4\end{array}$ & $\begin{array}{l}\text { Máximo } \\
\text { art. } 19\end{array}$ & - & $\begin{array}{l}\text { Cortes de } \\
\text { Aragón }\end{array}$ & No \\
\hline Asturias & $\begin{array}{l}\text { L.O. } 7 / 1981,30 \text { dic. } \\
\text { (BOE } 9,11-1-82)\end{array}$ & $\begin{array}{l}35 / 45 \\
\text { art. } 25.2\end{array}$ & $\begin{array}{l}\text { Proporcional } \\
\text { art. } 25.1\end{array}$ & - & - & - & $\begin{array}{l}\text { Junta General } \\
\text { del P. de Ast. }\end{array}$ & No \\
\hline Baleares & $\begin{array}{l}\text { L.O. } 22 / 1983,28 \text { feb. } \\
\text { (BOE } 51,1-3-83 \text { ) }\end{array}$ & - & $\begin{array}{l}\text { Proporcional } \\
\text { art. } 20.1\end{array}$ & $\begin{array}{l}\text { Territorialidad } \\
\text { art. } 20.1\end{array}$ & - & - & $\begin{array}{l}\text { Parl. de las } \\
\text { Islas Baleares }\end{array}$ & No \\
\hline Canarias & $\begin{array}{l}\text { L.O. 10/1982, } 10 \text { agos. } \\
\text { (BOE 195, 16-8-82) }\end{array}$ & $\begin{array}{l}50 / 70 \\
\text { art. } 8.3\end{array}$ & $\begin{array}{l}\text { Proporcional } \\
\text { art. } 8.2\end{array}$ & $\begin{array}{l}\text { Insulares } \\
\text { art. } 8.4\end{array}$ & $\begin{array}{l}\text { Fija } \\
\text { Trans. } 1 .^{a}\end{array}$ & $\begin{array}{l}3 \mathrm{R} / 20 \mathrm{D} \\
\text { art. } 8.2\end{array}$ & $\begin{array}{l}\text { Parlamento } \\
\text { Canario }\end{array}$ & No \\
\hline Cantabria & $\begin{array}{l}\text { L.O. } 8 / 1981,30 \text { dic. } \\
\text { (BOE } 9,11-1-82)\end{array}$ & $\begin{array}{l}35 / 45 \\
\text { art. } 10.4\end{array}$ & $\begin{array}{l}\text { Proporcional } \\
\text { art. } 10.1\end{array}$ & $\begin{array}{l}\text { Único } \\
\text { art. } 10.2\end{array}$ & - & - & $\begin{array}{l}\text { Asamb. Region. } \\
\text { de Cantabria }\end{array}$ & No \\
\hline Castilla y León & $\begin{array}{l}\text { L.O. 4/1983, } 25 \text { feb. } \\
\text { (BOE 52, 2-3-83) }\end{array}$ & - & $\begin{array}{l}\text { Proporcional } \\
\text { art. } 10.1\end{array}$ & $\begin{array}{l}\text { Provinciales } \\
\text { art. } 10.2\end{array}$ & $\begin{array}{l}3+1 / 45.000 \\
\text { art. } 10.2\end{array}$ & - & $\begin{array}{l}\text { Cortes de Castilla } \\
\text { y León }\end{array}$ & No \\
\hline Castilla-La Mancha & $\begin{array}{l}\text { L.O. 9/1982, } 10 \text { agos. } \\
\text { (BOE 195, 16-8-82) }\end{array}$ & $\begin{array}{l}40 / 50 \\
\text { art. } 10.1\end{array}$ & $\begin{array}{l}\text { Proporcional } \\
\text { art. } 10.2\end{array}$ & $\begin{array}{l}\text { Provinciales } \\
\text { art. } 10.2\end{array}$ & - & - & $\begin{array}{l}\text { Cortes de Castilla- } \\
\text { La Mancha }\end{array}$ & No \\
\hline Cataluña & $\begin{array}{l}\text { L.O. 4/1979, } 18 \text { dic. } \\
\text { (BOE 306, 22-12-79) }\end{array}$ & $\begin{array}{l}135 \\
\text { Trans. } 4 .^{\mathrm{a}}\end{array}$ & $\begin{array}{l}\text { Proporcional } \\
\text { art. } 31.1\end{array}$ & $\begin{array}{l}\text { Provinciales } \\
\text { Trans. } 4 .^{a}\end{array}$ & $\begin{array}{l}\text { Máx. y Mín. } \\
1 / 40.000 \\
1 / 50.000 \\
\text { Trans. } 4 .^{a}\end{array}$ & - & $\begin{array}{l}\text { Parlamento de } \\
\text { Cataluña }\end{array}$ & Sí \\
\hline
\end{tabular}


TABLA 1 (continuación)

Elementos del sistema electoral contenidos en los Estatutos de Autonomía

\begin{tabular}{|c|c|c|c|c|c|c|c|c|}
\hline Comunidad & $\begin{array}{l}\text { Referencia } \\
\text { legislativa }\end{array}$ & $\begin{array}{c}\text { Tamaño } \\
\text { Parlamento }\end{array}$ & $\begin{array}{l}\text { Fórmula } \\
\text { escrutinio }\end{array}$ & $\begin{array}{l}\text { Delimitación } \\
\text { distritos }\end{array}$ & $\begin{array}{l}\text { Magnitud } \\
\text { distritos }\end{array}$ & $\begin{array}{l}\text { Barreras } \\
\text { minimas }\end{array}$ & $\begin{array}{l}\text { Denominación } \\
\text { Parlamento }\end{array}$ & $\begin{array}{l}\text { Disolución } \\
\text { anticipada }\end{array}$ \\
\hline $\begin{array}{l}\text { Comunidad } \\
\text { Valenciana }\end{array}$ & $\begin{array}{l}\text { L.O. 5/1982, } 1 \text { jul. } \\
\text { (BOE } 164,10-7-82)\end{array}$ & $\begin{array}{l}75 / 100 \\
\text { art. } 12.1\end{array}$ & $\begin{array}{l}\text { Proporcional } \\
\text { art. } 12.1\end{array}$ & $\begin{array}{l}\text { Comarcales } \\
\text { art. } 12.1\end{array}$ & $\begin{array}{l}\text { Máx. y Mín. } \\
\text { 20+ Prop. Dem. } \\
\text { art. } 13\end{array}$ & $\begin{array}{l}5 \mathrm{R} \\
\text { art. } 12.2\end{array}$ & $\begin{array}{l}\text { Cortes } \\
\text { Valencianas }\end{array}$ & No \\
\hline Extremadura & $\begin{array}{l}\text { L.O. 1/1983, } 25 \text { feb. } \\
\text { (BOE 49, 26-2-83) }\end{array}$ & $\begin{array}{l}65 \text { Máx. } \\
\text { art. } 22.1\end{array}$ & $\begin{array}{l}\text { Proporcional } \\
\text { art. } 22.1\end{array}$ & $\begin{array}{l}\text { Provinciales } \\
\text { art. } 22.2\end{array}$ & $\begin{array}{l}\text { Mín. + Dem. } \\
\text { art. } 22.2\end{array}$ & - & $\begin{array}{l}\text { Asamblea de } \\
\text { Extremadura }\end{array}$ & No \\
\hline Galicia & $\begin{array}{l}\text { L.O. 1/1981, } 6 \text { abr. } \\
\text { (BOE 101, 28-4-81) }\end{array}$ & $\begin{array}{l}60 / 80 \\
\text { art. } 11.5\end{array}$ & $\begin{array}{l}\text { Proporcional } \\
\text { art. } 11.2\end{array}$ & $\begin{array}{l}\text { Provinciales } \\
\text { art. } 11.4\end{array}$ & - & - & $\begin{array}{l}\text { Parlamento de } \\
\text { Galicia }\end{array}$ & Sí \\
\hline Madrid & $\begin{array}{l}\text { L.O. 3/1983, } 25 \text { feb. } \\
\text { (BOE 51, 1-3-83) }\end{array}$ & $\begin{array}{l}1 / 50.000 \\
\text { art. } 10.2\end{array}$ & $\begin{array}{l}\text { Proporcional } \\
\text { art. } 11.3\end{array}$ & $\begin{array}{l}\text { Único } \\
\text { art. } 11.1\end{array}$ & - & $\begin{array}{l}5 \mathrm{D} / \mathrm{R} \\
\text { art. } 11.4\end{array}$ & $\begin{array}{l}\text { Asamblea de } \\
\text { Madrid }\end{array}$ & No \\
\hline Murcia & $\begin{array}{l}\text { L.O. 4/1982, } 9 \text { jun. } \\
\text { (BOE } 146,19-6-82 \text { ) }\end{array}$ & $\begin{array}{l}35 / 45 \\
\text { art. } 24.2\end{array}$ & $\begin{array}{l}\text { Proporcional } \\
\text { art. } 24.1\end{array}$ & - & - & - & $\begin{array}{l}\text { Asambela Reg. } \\
\text { de Murcia }\end{array}$ & No \\
\hline Navarra & $\begin{array}{l}\text { L.O. 13/1982, } 10 \text { agos. } \\
\text { (BOE 195, 16-8-82) }\end{array}$ & $\begin{array}{l}40 / 60 \\
\text { art. } 15.2\end{array}$ & $\begin{array}{l}\text { Proporcional } \\
\text { art. } 15.2\end{array}$ & - & - & - & $\begin{array}{l}\text { Parl. o Cortes } \\
\text { de Navarra }\end{array}$ & No \\
\hline País Vasco & $\begin{array}{l}\text { L.O. } 3 / 1979,18 \text { dic. } \\
\text { (BOE } 306,22-12-79 \text { ) }\end{array}$ & - & $\begin{array}{l}\text { Proporcional } \\
\text { art. } 26.3\end{array}$ & $\begin{array}{l}\text { Provinciales } \\
\text { art. } 26.2\end{array}$ & $\begin{array}{l}\text { Iguales } \\
\text { art. } 26.1\end{array}$ & - & $\begin{array}{l}\text { Parlamento } \\
\text { Vasco }\end{array}$ & Sí \\
\hline La Rioja & $\begin{array}{l}\text { L.O. } 3 / 1982,9 \text { jun. } \\
\text { (BOE } 146,19-6-82)\end{array}$ & $\begin{array}{l}32 / 40 \\
\text { art. } 18.1\end{array}$ & $\begin{array}{l}\text { Proporcional } \\
\text { art. } 18.3\end{array}$ & $\begin{array}{l}\text { Único } \\
\text { art. } 18.2\end{array}$ & - & - & $\begin{array}{l}\text { Diput. Gral. de } \\
\text { La Rioja }\end{array}$ & No \\
\hline
\end{tabular}

FUENTE: Elaboración propia. 
común. Por un lado, por el papel clave que han adquirido los partidos nacionalistas mayoritarios vascos y catalanes en la gobernabilidad del Estado; y, por otro, por ser Galicia y Andalucía, respectivamente, los graneros electorales de los dos partidos mayoritarios sobre cuya alternancia descansa dicha gobernabilidad. Al mismo tiempo, el uso estratégico de esa especie de autorregulación del ritmo de convocatoria puede tener una incidencia mucho mayor en las elecciones legislativas cercanas en el tiempo sobre el sistema político nacional (Caciagli y Corbetta, 1987), como hemos podido comprobar en los últimos años. Desde otro punto de vista, la homogeneización y simultaneidad de las elecciones municipales y regionales de las autonomías de régimen común, a la vez que racionalizan el proceso electoral, contribuyen a reducir el valor político de sus resultados, convirtiéndolas en elecciones de segundo orden. En este sentido, es relevante la hipótesis de Vallès (1987) sobre el interés de los grandes partidos nacionales de actuar con ventaja en este tipo de elecciones, tratando de limitar la importancia que pudieran adquirir los partidos regionales; una estrategia para la que han encontrado aliados en los principales partidos nacionalistas de las Comunidades históricas, interesados, a su vez, en diferenciar claramente su estatus político de autogobierno.

Todos los Estatutos hacen referencia genérica a la fórmula de escrutinio de carácter proporcional sin más concreción, a excepción de Andalucía, que la identifica con la vigente para el Congreso de los Diputados. La mayoría, con las excepciones de Baleares, Castilla y León y el País Vasco, cuantifican los límites de su tamaño parlamentario. Igualmente, casi todos definen el criterio de delimitación de sus distritos o circunscripciones, excluyéndose de esta norma Asturias, Baleares, Comunidad Valenciana, Murcia y Navarra. Algunas Comunidades van más allá e incluyen en sus Estatutos algunos criterios de cálculo de las magnitudes de sus distritos (Canarias, Castilla y León, Cataluña, Comunidad Valenciana, Extremadura y País Vasco) o sus límites mínimo y/o máximo (Andalucía y Aragón), de una forma más o menos concreta. Finalmente, sólo Canarias, Comunidad Valenciana y Madrid regulan en sus respectivos Estatutos de Autonomía las barreras mínimas de votos para acceder al reparto de escaños. Decíamos más arriba que excluíamos de este análisis los elementos restantes del sistema electoral incluidos en las disposiciones transitorias, precisamente por tener este carácter, a pesar de que muchos de ellos prefiguraron el sistema electoral definitivo en la medida en que fueron luego consagrados en las respectivas leyes electorales.

Comenzando por el País Vasco en 1983 y con la única excepción de Cataluña, todas las Comunidades Autónomas promulgan sus leyes electorales entre 1985 (Galicia) y 1991 (La Rioja), en las que incluyen un título específico, completando la institucionalización de todos los elementos de sus respectivos sistemas electorales, tal como son sistematizados en la tabla 2. Sólo Cataluña (en 1984) y Canarias (en 1987) se limitan a referirse a los Estatutos respectivos y a la adecuación de las normas electorales generales contenidas en el R.D. 20/1977, de 18 de marzo, la primera, y en la LOREG, la segunda. De este 
TABLA 2

Elementos del sistema electoral desarrollados por las leyes electorales de las CC.AA.

\begin{tabular}{|c|c|c|c|c|c|c|}
\hline Comunidad & Referencia legislativa & Tamaño Parlamento & Fórmula escrutinio & Delimitación distritos & s Magnitud distritos & Barreras minimas \\
\hline Andalucía & $\begin{array}{l}\text { L. 1/1986, } 2 \text { enero } \\
\text { BOJA 3, 14-1-86 (Tít. IV) }\end{array}$ & $\begin{array}{l}109 \\
\text { art. } 17.1\end{array}$ & $\begin{array}{l}\text { D'Hondt } \\
\text { art. 18.1.b-e }\end{array}$ & Estatuto & $\begin{array}{l}8+\text { Dem. } \\
\text { art. } 17.2 \text { y } 3\end{array}$ & $\begin{array}{l}3 \mathrm{D} \\
\text { art. 18.1.a }\end{array}$ \\
\hline Aragón & $\begin{array}{l}\text { L. 2/1987, } 16 \text { febrero } \\
\text { BOA 19, 18-2-87 (Tít. IV) }\end{array}$ & $\begin{array}{l}67 \\
\text { art. } 13.1\end{array}$ & $\begin{array}{l}\text { D'Hondt } \\
\text { art. 14.b-e }\end{array}$ & Estatuto & $\begin{array}{l}13+\text { Dem. } \\
\text { art. } 13.2 \text { y } 3\end{array}$ & $\begin{array}{l}3 \mathrm{D} \\
\text { art. } 14 . \mathrm{a}\end{array}$ \\
\hline Asturias & $\begin{array}{l}\text { L. 14/1986, } 26 \text { diciembre } \\
\text { BOPA 9, 13-1-87 (Tít. III) }\end{array}$ & $\begin{array}{l}45 \\
\text { art. } 12\end{array}$ & $\begin{array}{l}\text { D'Hondt } \\
\text { art. 13.b-e }\end{array}$ & $\begin{array}{l}\text { Comarcales } \\
\text { art. } 10\end{array}$ & $\begin{array}{l}2+\text { Dem. } \\
\text { art. } 12\end{array}$ & $\begin{array}{l}3 \mathrm{D} \\
\text { art. 13.a }\end{array}$ \\
\hline Baleares & $\begin{array}{l}\text { L. 8/1986, } 26 \text { noviembre } \\
\text { BOE 30, 4-2-87 (Tít. IV) }\end{array}$ & $\begin{array}{l}59 \\
\text { art. } 12.1\end{array}$ & $\begin{array}{l}\text { art. } 163.1 \text { LOREG } \\
\text { art. } 12.3\end{array}$ & $\begin{array}{l}\text { Insulares } \\
\text { art. } 12.1\end{array}$ & $\begin{array}{l}\text { Fija } \\
\text { art. } 12.2\end{array}$ & $\begin{array}{l}5 \mathrm{D} \\
\text { art. } 12.4\end{array}$ \\
\hline Canarias & $\begin{array}{l}\text { L. } 3 / 1987,3 \text { abril } \\
\text { BOCA 41, 4-4-87 }\end{array}$ & $\begin{array}{l}(60) \\
\text { Estatuto }\end{array}$ & LOREG & Estatuto & Etatuto & Estatuto \\
\hline Cantabria & $\begin{array}{l}\text { L. 5/1987, } 27 \text { marzo } \\
\text { BOC 86, 10-4-87 (Tít. III) }\end{array}$ & $\begin{array}{l}39 \\
\text { art. } 17.1\end{array}$ & $\begin{array}{l}\text { art. } 163.1 \text { LOREG } \\
\text { art. } 17.2\end{array}$ & Estauto & - & $\begin{array}{l}5 \mathrm{D} / \mathrm{R} \\
\text { art. } 17.3\end{array}$ \\
\hline Castilla y León & $\begin{array}{l}\text { L. } 3 / 1987,30 \text { marzo } \\
\text { BOCL 40, 1-4-87 (Tít. IV) }\end{array}$ & Estatuto & $\begin{array}{l}\text { D'Hondt } \\
\text { art. 20.b-e }\end{array}$ & Estatuto & Estatuto & $\begin{array}{l}3 \mathrm{D} \\
\text { art. 20.a }\end{array}$ \\
\hline Castilla-La Mancha & $\begin{array}{l}\text { L. 5/1986, } 23 \text { diciembre } \\
\text { DOCM 1, 5-1-87 (Tít. III) }\end{array}$ & $\begin{array}{l}47 \\
\text { art. } 16.1\end{array}$ & $\begin{array}{l}\text { D'Hondt } \\
\text { art. 17.b-d }\end{array}$ & Estatuto & $\begin{array}{l}5+\text { Dem. } \\
\text { art. } 16.2 \text { y } 3\end{array}$ & $\begin{array}{l}3 \mathrm{D} \\
\text { art. 17.a }\end{array}$ \\
\hline Cataluña* & $\begin{array}{l}\text { L. 5/1984, } 5 \text { marzo } \\
\text { DOGC 414, 7-3-84 }\end{array}$ & Estatuto & $\begin{array}{l}\text { R.D. } 20 / 1977 \\
18 \text { marzo }\end{array}$ & Estatuto & Estatuto & $\begin{array}{l}3 \text { D } \\
\text { R.D. } 20 / 1977,18 \text { marzo }\end{array}$ \\
\hline
\end{tabular}


TABLA 2 (continuación)

Elementos del sistema electoral desarrollados por las leyes electorales de las CC.AA.

\begin{tabular}{|c|c|c|c|c|c|c|}
\hline Comunidad & Referencia legislativa & Tamaño Parlamento & Fórmula escrutinio & Delimitación distritos & os Magnitud distritos & Barreras minimas \\
\hline $\begin{array}{l}\text { Comunidad } \\
\text { Valenciana }\end{array}$ & $\begin{array}{l}\text { L. 1/1987, } 31 \text { marzo } \\
\text { DOGV 561, 6-4-87 (Tít. II) }\end{array}$ & $\begin{array}{l}89 \\
\text { art. } 11.1\end{array}$ & $\begin{array}{l}\text { D'Hondt } \\
\text { art. 12.b-e }\end{array}$ & $\begin{array}{l}\text { Provinciales } \\
\text { art. } 10\end{array}$ & $\begin{array}{l}20+\text { D'Hondt y dem. } \\
\text { art. } 11.3\end{array}$ & Estatuto \\
\hline Extremadura & $\begin{array}{l}\text { L. 2/1987, } 16 \text { marzo } \\
\text { BOE 3, 16-3-87 (Tít. III) }\end{array}$ & $\begin{array}{l}65 \\
\text { art. } 18.1\end{array}$ & $\begin{array}{l}\text { D'Hondt } \\
\text { art. 19.1.b-e }\end{array}$ & Estatuto & $\begin{array}{l}20+\text { Dem. } \\
\text { art. } 18.2 \text { y } 3\end{array}$ & $\begin{array}{l}5 \mathrm{D} / \\
\text { Cand. Reg y 5R art. 19.1.a }\end{array}$ \\
\hline Galicia** & $\begin{array}{l}\text { L. } 8 / 1985,13 \text { agosto } \\
\text { DOG } 156,16-8-85 \text { (Tít. II) }\end{array}$ & $\begin{array}{l}65 \\
\text { art. } 9.1\end{array}$ & $\begin{array}{l}\text { D'Hondt } \\
\text { art. 10.b-e }\end{array}$ & Estatuto & $\begin{array}{l}10+\text { Dem. } \\
\text { art. } 9.2 \text { y } 3\end{array}$ & $\begin{array}{l}5 \mathrm{D}(3 \mathrm{D}) \\
\text { art. } 10 . \mathrm{a}\end{array}$ \\
\hline Madrid & $\begin{array}{l}\text { L. } 11 / 1986,16 \text { diciembre } \\
\text { BOE } 41,17-2-87 \text { (Cap. VII) }\end{array}$ & Estatuto & $\begin{array}{l}\text { art. } 163.1 \text { LOREG } \\
\text { art. } 18.3\end{array}$ & Estatuto & - & Estatuto \\
\hline Murcia & $\begin{array}{l}\text { L. 2/1987, } 24 \text { febrero } \\
\text { BORM 59, 12-3-87 (Tít. III) }\end{array}$ & $\begin{array}{l}45 \\
\text { art. } 14.1\end{array}$ & $\begin{array}{l}\text { D'Hondt } \\
\text { art. 15.b-e }\end{array}$ & $\begin{array}{l}\text { Comarcales } \\
\text { art. } 13\end{array}$ & $\begin{array}{l}5+\text { Dem. } \\
\text { art. } 14.2 \text { y } 3\end{array}$ & $\begin{array}{l}5 \mathrm{R} \\
\text { art. } 15 . \mathrm{a}\end{array}$ \\
\hline Navarra & $\begin{array}{l}\text { L.F. } 16 / 1986,17 \text { noviembre } \\
\text { BOE } 17,20-1-87 \text { (Tít. V) }\end{array}$ & $\begin{array}{l}50 \\
\text { art. } 1\end{array}$ & $\begin{array}{l}\text { art. 163.1 LOREG } \\
\text { art. } 10.2\end{array}$ & $\begin{array}{l}\text { Único } \\
\text { art. } 9\end{array}$ & - & $\begin{array}{l}3 \mathrm{D} / \mathrm{R} \\
\text { art. } 10.1\end{array}$ \\
\hline País Vasco*** & $\begin{array}{l}\text { L. 5/1990, } 15 \text { junio } \\
\text { BOPV 134, 6-7-90 (Tít. II) }\end{array}$ & $\begin{array}{l}75 \\
\text { art. } 10\end{array}$ & $\begin{array}{l}\text { D'Hondt } \\
\text { art. 12.1.b-f }\end{array}$ & Estatuto & $\begin{array}{l}25 \\
\text { art. } 10\end{array}$ & $\begin{array}{l}5 \mathrm{D} \\
\text { art. } 11.1\end{array}$ \\
\hline La Rioja & $\begin{array}{l}\text { L. 3/1991, } 21 \text { marzo } \\
\text { BOE 74, 27-3-91 (Tít. III) }\end{array}$ & $\begin{array}{l}33 \\
\text { art. } 19\end{array}$ & $\begin{array}{l}\text { D'Hondt } \\
\text { art. 20.b-e }\end{array}$ & Estatuto & - & $\begin{array}{l}5 \mathrm{D} / \mathrm{R} \\
\text { art. } 20 . \mathrm{a}\end{array}$ \\
\hline
\end{tabular}

* Cataluña no tiene propiamente una Ley Electoral, sino que se limita en un artículo único a remitirse a la Disp. Transitoria 4. ${ }^{a}$ del Estatuto y al R.D. $20 / 1977$, de 18 de marzo.

** Reformado por L. 15/1992, de 30 de diciembre (BOE 46, 23-2-93), elevando el 3 por 100 anterior.

*** El País Vasco fue el primero en promulgar una Ley Electoral: L. 28/1983, de 25 de noviembre (BOPV 182, 10-12-83).

FUENTE: Elaboración propia. 
modo, salvo Castilla y León, que ya lo había hecho en su Estatuto, todas las demás Comunidades Autónomas concretan el número exacto de sus parlamentarios. La homogeneidad y unanimidad es total, aunque la forma textual varíe, en la adopción de la fórmula de escrutinio proporcional de media más elevada según la variante D'Hondt, imperante para las elecciones legislativas y locales. Las que no lo habían hecho en sus Estatutos (Asturias, Baleares, Comunidad Valenciana, Murcia y Navarra), determinan en las leyes electorales los criterios de delimitación de sus circunscripciones. Lo mismo sucede con las once que no habían cuantificado la magnitud de sus distritos en el Estatuto, haciéndolo posteriormente en la respectiva ley electoral. Finalmente, exceptuando Canarias, Comunidad Valenciana y Madrid, que ya habían fijado las barreras mínimas de votos, las catorce restantes lo hacen en sus leyes electorales o se remiten a la normativa general, como es el caso de Cataluña.

\section{LA TALLA Y EL COEFICIENTE DE REPRESENTACIÓN DE LOS PARLAMENTOS AUTÓNOMOS}

La talla de las asambleas legislativas es la primera pieza de la representación, básicamente porque según sea alta o baja está afectada por el carácter probabilístico de su representatividad estadística, de manera que lo más frecuente es que, de no cumplirse unos mínimos numéricos elevados, condicione la proporcionalidad básica del sistema de representación. El segundo componente de la representación tiene un carácter cualitativo y viene definido por el número de electores potenciales que, por término medio, puede representar cada parlamentario, tal como lo cuantifica el coeficiente electoral al dividir el censo electoral por el total de representantes. La hipótesis es que un coeficiente bajo puede añadir a las condiciones probabilísticas de la representatividad estadística de una talla alta la cualidad de una potencial mayor cercanía entre cada diputado y sus ciudadanos, mientras que una ratio mayor puede empeorar tales condiciones.

En la tabla 3 mostramos el cálculo de tales parámetros para todas las Comunidades Autónomas en función de sus respectivas tallas censales. Tomando como referencia los censos electorales de cada una de ellas en la última elección autonómica producida entre 1993 (Galicia) y 1996 (Andalucía), ya nos dan idea de la gran oscilación existente en los pesos demográficos de las distintas Comunidades, que hacen que la mayor (Andalucía) multiplique por 25 la demografía de la menor (La Rioja). En total, las 17 Comunidades Autónomas producen un tejido representativo de 1.180 diputados regionales, con un coeficiente electoral medio de un diputado por cada 26.872 electores o, en términos físicos, un diputado por cada $428 \mathrm{~km}^{2}$, que, en principio, definen una alta cobertura representativa, a añadir a los 350 diputados y más de 250 senadores de las Cortes Generales. 


\section{TABLA 3}

Tamaños demográficos y parlamentarios de las CC.AA., 1993-1996

\begin{tabular}{|c|c|c|c|}
\hline & Censo electoral & Escaños & $\begin{array}{c}\text { Coeficiente } \\
\text { electoral* }\end{array}$ \\
\hline 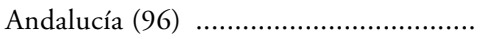 & 5.511 .207 & 109 & 50.561 \\
\hline 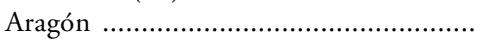 & 983.616 & 67 & 14.681 \\
\hline 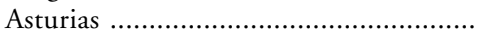 & 944.949 & 45 & 20.999 \\
\hline 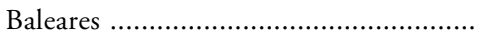 & 591.231 & 59 & 10.021 \\
\hline 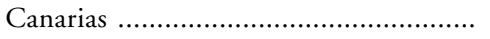 & 1.231 .900 & 60 & 20.532 \\
\hline 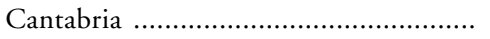 & 435.752 & 39 & 11.173 \\
\hline 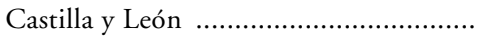 & 2.080 .227 & 84 & 24.765 \\
\hline 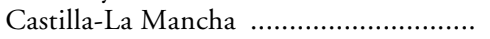 & 1.352 .958 & 47 & 28.786 \\
\hline 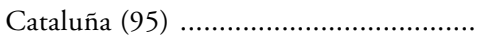 & 5.029 .727 & 135 & 37.257 \\
\hline 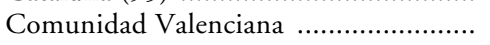 & 3.093 .574 & 89 & 34.759 \\
\hline 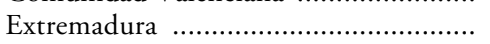 & 836.007 & 65 & 12.862 \\
\hline 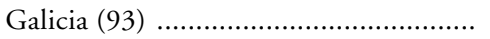 & 2.293 .169 & 75 & 30.575 \\
\hline 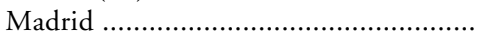 & 4.081 .929 & 103 & 39.630 \\
\hline 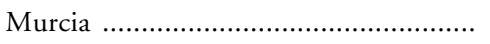 & 837.180 & 45 & 18.604 \\
\hline 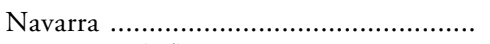 & 437.776 & 50 & 8.755 \\
\hline 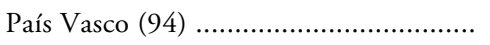 & 1.749 .250 & 75 & 23.323 \\
\hline 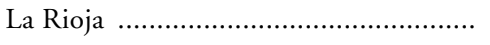 & 218.519 & 33 & 6.622 \\
\hline 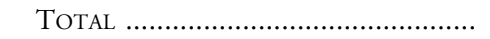 & 31.708 .971 & 1.180 & 26.872 \\
\hline
\end{tabular}

* Número de electores por escaño, que resulta de dividir el censo electoral por el total de escaños.

FUENTE: Juntas Electorales y elaboración propia.

La talla parlamentaria tiene un recorrido que va de la mínima de La Rioja con 33 diputados a la máxima de Cataluña con 135 escaños, que, en todo caso, reducen el efecto multiplicador anterior de 25 a 4 veces. Teniendo en cuenta que la talla media es de unos 69 diputados por asamblea, podemos clasificar las Comunidades Autónomas en tres tipos según su talla sea alta (más de 100 escaños), media (entre 50 y 90) y baja (por debajo de 60). Así: en el primer tipo estarían Cataluña, Andalucía y Madrid, que son las que tienen un mayor volumen demográfico; Cataluña, a pesar de su menor extensión territorial y peso demográfico, supera a Andalucía en una cuarta parte de su talla parlamentaria y, mientras la primera tiene un diputado por cada $236 \mathrm{~km}^{2}$, la segunda lo tiene por cada $800 \mathrm{~km}^{2}$, situándose Madrid en sólo $77 \mathrm{~km}^{2}$, que es el mínimo de toda España, por su carácter uniprovincial y el grado de concentración urbana. En el segundo tipo estarían la Comunidad Valenciana, Castilla y León, Galicia, País Vasco, Aragón, Extremadura, Canarias y Baleares, oscilando su cobertura territorial entre el mínimo de Baleares $\left(85 \mathrm{~km}^{2}\right.$ por diputa- 
do) y el máximo de Castilla y León $\left(1.121 \mathrm{~km}^{2}\right.$ por diputado), sin que aparezcan en este tipo regiones uniprovinciales. El tercer tipo incluiría a las seis restantes (Castilla-La Mancha, Navarra, Asturias, Murcia, Cantabria y La Rioja), todas ellas por debajo de 50 diputados y todas las uniprovinciales, con la excepción ya citada de Madrid, siendo su cobertura territorial la que más recorrido tiene, al oscilar entre el mínimo de Cantabria $\left(136 \mathrm{~km}^{2}\right.$ por diputado) y el máximo de toda España, detentado por Castilla-La Mancha $\left(1.686 \mathrm{~km}^{2}\right.$ por diputado).

El recorrido del coeficiente electoral de las distintas Comunidades Autónomas se correlaciona casi perfectamente con su posición respectiva en el ránking demográfico, oscilando entre el mínimo de La Rioja (uniprovincial y la más pequeña demográfica y territorialmente, junto con Cantabria), con 6.622 electores por diputado, y el máximo de Andalucía (la que comprende mayor número de circunscripciones provinciales y con mayor talla demográfica y física), con 50.561 electores por representante, que reduce el efecto multiplicador censal del 25 al 7,6. Si tomamos en cuenta la media para el conjunto de las Comunidades Autónomas, que se sitúa en 26.872 electores por diputado, podemos clasificarlas en dos grupos: el primero, situado por encima de dicho promedio, lo forman, por este orden, Andalucía, Madrid, Cataluña, Comunidad Valenciana, Galicia y Castilla-La Mancha; el segundo, por debajo del mismo, lo engrosan las once restantes (Castilla y León, País Vasco, Asturias, Canarias, Murcia, Aragón, Extremadura, Cantabria, Baleares, Navarra y La Rioja).

Sabido es que la decisión política sobre las tallas parlamentarias no se orienta, necesaria ni principalmente, por criterios técnicos de representatividad o proporcionalidad, ni siquiera por un único criterio, sino que en ella se ponderan distintas razones, entre las que la presupuestaria y funcional suelen pesar por encima de la demográfica o la territorial. Con todo y a pesar de la fragmentación del sistema, el conjunto, como se puede comprobar, resulta altamente coherente y bastante racional, pudiendo tomarse en consideración la posibilidad de proceder a una ligera elevación de las tallas parlamentarias de algunas Comunidades Autónomas como Andalucía, Asturias, Canarias o Castilla-La Mancha.

\section{LOS DISTRITOS ELECTORALES: DESIGUALDAD Y DESPROPORCIONALIDAD}

La delimitación y el tamaño de los distritos electorales es clave en la evaluación de los efectos políticos y en el estudio de los rendimientos de los sistemas electorales, tal como han planteado, entre otros, Duverger (1965), Rae (1971), Sartori (1984 y 1986), Lijphart (1990) y Nohlen (1981a y 1991). Es conocida también la coincidencia de los estudiosos (Vallès, 1982; Nohlen, 1983; Montero, Llera y Torcal, 1992) en identificar la magnitud de los distritos como la principal fuente de desigualdad y desproporcionalidad del sistema electoral español. 
De acuerdo con la estructura electoral española, todas las circunscripciones o distritos electorales son plurinominales, lo cual es válido también para el nivel autonómico, con la única excepción de la isla de Formentera, como hemos visto en el primer apartado. Siguiendo la pauta general, como se puede comprobar en la tabla 4, la provincia vuelve a ser el criterio básico de delimitación de los distritos electorales. De este modo, podemos clasificar las Comunidades Autónomas en cuatro tipos: el primero y mayoritario es el de las pluriprovinciales, que reproducen a nivel autonómico su sistema de distritos provinciales del ámbito legislativo general (Andalucía, Aragón, Galicia, Castilla y León, Castilla-La Mancha, Cataluña, Comunidad Valenciana, Extremadura y País Vasco); el segundo tipo, también numeroso, es el de las uniprovinciales de distrito único o regional (Cantabria, Madrid, Navarra y La Rioja, a las que habría que unir ahora Ceuta y Melilla); el tercer tipo corresponde a las insulares (Baleares y Canarias), que convierten a todas y cada una de sus islas en distritos electorales; y el cuarto tipo es el de las dos uniprovinciales (Asturias y Murcia), que segmentan sus regiones en tres y cinco distritos zonales o comarcales, respectivamente.

\section{TABLA 4}

Delimitación, magnitud de los distritos y desigualdad de voto en las CC.AA., 1993-1996

\begin{tabular}{|c|c|c|c|c|}
\hline & Distritos (1) & Magnitud (2) & Desigualdad (3) & Evolución (4) \\
\hline Andalucía (96) ............................... & $\mathrm{P}(8)$ & $11 / 19$ & 2,14 & $=$ \\
\hline 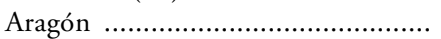 & $P(3)$ & $16 / 33$ & 2,89 & + \\
\hline 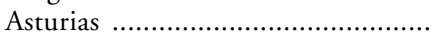 & $Z(3)$ & $5 / 33$ & 1,58 & $=$ \\
\hline 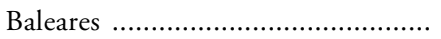 & I (4) & $1 / 33$ & 3,91 & $=$ \\
\hline 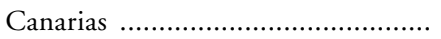 & I (7) & $3 / 15$ & 17,48 & - \\
\hline 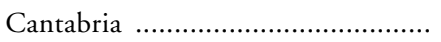 & $\mathrm{U}$ & 39 & - & \\
\hline Castilla y León & $\mathrm{P}(9)$ & $5 / 15$ & 1,45 & - \\
\hline Castilla-La Mancha $\ldots \ldots \ldots \ldots \ldots \ldots \ldots \ldots$ & $\mathrm{P}(5)$ & $7 / 11$ & 1,87 & $=$ \\
\hline 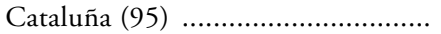 & $\mathrm{P}(4)$ & $15 / 85$ & 2,31 & $=$ \\
\hline Comunidad Valenciana ................. & $\mathrm{P}(3)$ & $22 / 37$ & 2,47 & - \\
\hline 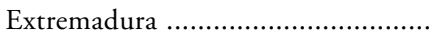 & $\mathrm{P}(2)$ & $30 / 35$ & 1,24 & $=$ \\
\hline 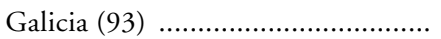 & $\mathrm{P}(4)$ & $15 / 24$ & 1,84 & + \\
\hline 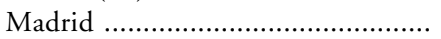 & $\mathrm{U}$ & 103 & - & \\
\hline Murcia & $Z(5)$ & $3 / 21$ & 1,64 & $=$ \\
\hline 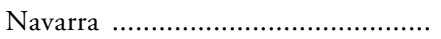 & $\mathrm{U}$ & 50 & - & \\
\hline 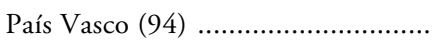 & $\mathrm{P}(3)$ & 25 & 4,2 & $=$ \\
\hline La Rioja & $\mathrm{U}$ & 33 & - & \\
\hline
\end{tabular}

(1) $\mathrm{P}=$ provinciales; $\mathrm{Z}$ = zonales; $\mathrm{I}$ = insulares; $\mathrm{U}=$ únicos.

(2) Recorrido entre el número de escaños de los distritos menor y mayor.

(3) Ratio entre los coeficientes electorales de los distritos mayor y menor.

(4) Evolución del ratio de desigualdad en relación al período 1989-1992.

FUENTE: Elaboración propia. 
Sin embargo, el aspecto más importante del sistema de distritos no es tanto su delimitación, más o menos justificada políticamente, cuanto el cálculo y la asignación de su magnitud, que es la verdadera causa de las distorsiones sobre la igualdad y la desproporcionalidad del voto. Si excluimos las cuatro Comunidades Autónomas de distrito único que, automáticamente, anulan tal efecto, al resto las podemos clasificar en tres grupos por sus criterios de cálculo, así: el primer grupo de seis (Asturias, Castilla y León, Castilla-La Mancha, Extremadura, Galicia y Murcia) que asigna un número mínimo de diputados a cada distrito y el resto los distribuye según criterios de proporcionalidad pura (resto mayor) en función de la población de derecho, con la excepción de Castilla y León, que aplica una ratio de 1 por cada 45.000 habitantes para los diputados sobrantes; el segundo grupo de tres (Andalucía, Aragón y la Comunidad Valenciana), además de hacer básicamente lo mismo que las anteriores, ponen un límite máximo a la desproporcionalidad entre provincias, con la peculiaridad de la Comunidad Valenciana, que aplica la fórmula D'Hondt para el reparto demográfico de los escaños sobrantes; el tercer grupo, formado por Cataluña, País Vasco y las Comunidades insulares, lo caracterizaremos como especial, ya que estas últimas asignan un número fijo y directo a cada isla sin mayor explicación; el País Vasco, basándose en razones históricas de foralidad, atribuye a cada provincia un número fijo e igual de escaños, y Cataluña representaría una variante del segundo grupo, en tanto en cuanto pone límites mínimo (para Girona, Lleida y Tarragona) y máximo (para Barcelona), repartiendo el resto según ratios diferenciales para las primeras (1 por cada 40.000) y para la segunda (1 por cada 50.000).

Como se puede comprobar, en lo fundamental se sigue aquí también la pauta nacional de combinar criterios territoriales y demográficos, limitando el efecto diferencial de estos últimos. Igualmente, la base de cálculo en la aplicación del criterio demográfico común es la población de derecho, como para el ámbito legislativo general, en lugar del censo electoral, lo cual significa que el padrón de habitantes (o, en el futuro inmediato, el registro de población) tiene mucha más incidencia electoral que el propio censo de electores. Este mecanismo distorsiona de forma generalizada la igualdad del voto, por el hecho de que tiende a privilegiar doblemente los distritos más deprimidos demográficamente (por su distinta estructura de edades), por un lado por la asignación territorial de escaños, pero, además, porque el criterio de la población de derecho beneficia a aquellos distritos que tienen menos electores por cada 100 habitantes, máxime si tenemos en cuenta que por nuestra peculiar dinámica demográfica, que afecta de forma diferencial a las distintas zonas urbanas o rurales, mientras que la población de derecho se ha incrementado desde 1977 en aproximadamente un 12 por 100, el censo electoral lo ha hecho en casi el 38 por 100 .

El primer efecto cuantificable, por tanto, de la magnitud de los distritos es la desigualdad del voto de los ciudadanos según donde voten. La comparación de los coeficientes electorales de los distritos mayor y menor de cada Comunidad y el cálculo de la correspondiente ratio de desigualdad, nos permite obser- 
var cómo el voto de un ciudadano canario de la isla de El Hierro vale 17,5 veces el de otro de Gran Canaria, el de un alavés cuatro veces el de un vizcaíno, casi lo mismo que el de un balear de la isla de Formentera en relación a otro elector mallorquín, el triple es lo que vale el voto de un turolense respecto de un zaragozano, y así sucesivamente, hasta el mínimo de 1,2 de Extremadura. La evolución, además, ha agravado este efecto en Aragón y Galicia, si bien lo ha reducido ligeramente en Canarias, Castilla-La Mancha y Comunidad Valenciana.

\section{TABLA 5}

Tipos de distritos de las Comunidades Autónomas por su magnitud, 1993-1996

\begin{tabular}{|c|c|c|c|c|c|c|}
\hline & $\begin{array}{c}I \\
\left(\begin{array}{ll}1 & \text { esc. }\end{array}\right)\end{array}$ & $\begin{array}{c}I I \\
(3-6 \text { esc.) }\end{array}$ & $\begin{array}{c}I I I \\
(7 / 8 \text { esc.) }\end{array}$ & $\begin{array}{c}I V \\
(+9 \text { esc. })\end{array}$ & $\begin{array}{c}\text { Umbral } \\
\text { prop. } \\
\text { (1) }\end{array}$ & $\begin{array}{l}\text { Dist. } \\
\text { Bip. } \\
\text { (2) }\end{array}$ \\
\hline Andalucía (96) ..................... & - & - & - & 8 & 100 & - \\
\hline 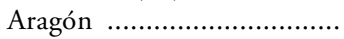 & - & - & - & 3 & 100 & - \\
\hline 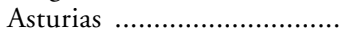 & - & 1 & 1 & 1 & 73 & $2 / 3$ \\
\hline 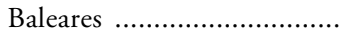 & $1(3)$ & - & 一 & 3 & 98 & - \\
\hline 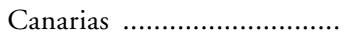 & - & 2 & 3 & 2 & 50 & $1 / 7$ \\
\hline 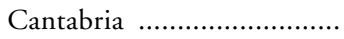 & - & - & 一 & 1 & 100 & - \\
\hline Castilla y León ..................... & - & 2 & 3 & 4 & 61 & $5 / 9$ \\
\hline Castilla-La Mancha .............. & - & - & 2 & 3 & 68 & $4 / 5$ \\
\hline Cataluña $(95)$....................... & - & - & - & 4 & 100 & - \\
\hline Comunidad Valenciana ...... & - & - & - & 3 & 100 & - \\
\hline Extremadura ........................... & - & - & - & 2 & 100 & - \\
\hline Galicia (93) ....................... & - & - & - & 4 & 100 & - \\
\hline 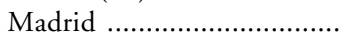 & - & - & - & 1 & 100 & - \\
\hline 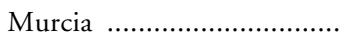 & - & 2 & 1 & 2 & 69 & $2 / 5$ \\
\hline 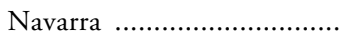 & - & - & - & 1 & 100 & - \\
\hline País Vasco (94) ...................... & - & - & - & 3 & 100 & - \\
\hline 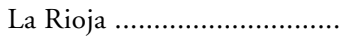 & - & - & - & 1 & 100 & - \\
\hline
\end{tabular}

(1) Porcentaje de escaños con menor desproporcionalidad.

(2) Número de distritos en los que sólo obtienen representación los dos principales partidos sobre el total de distritos.

(3) Distrito uninominal de Formentera.

FUENTE: Elaboración propia.

El segundo efecto conocido es el impacto de la magnitud de los distritos sobre la proporcionalidad. En la tabla 5 mostramos la conocida clasificación de los distritos según su magnitud. Obviamente, aquí se supera ampliamente el promedio de 6,7 diputados por circunscripción que sitúa a España entre los más bajos de Europa (Gallagher, 1991: 44), por lo que el impacto desproporcional por este factor a nivel autonómico es mucho más limitado que a nivel 
general. Si tenemos en cuenta que la proporcionalidad óptima (o desproporcionalidad mínima) sólo se puede obtener en distritos con 9 o más escaños, el impacto desproporcional de la baja magnitud de los distritos podría afectar en distinto grado al 50 por 100 de los escaños en Canarias, al 39 por 100 en Castilla y León, al 32 por 100 en Castilla-La Mancha, al 31 por 100 en Murcia, al 27 por 100 en Asturias y al 2 por 100 en Baleares, a la vista de lo que hemos denominado umbrales de proporcionalidad, que en total suponen 105 escaños o un 9 por 100 de los casi 1.200 escaños autonómicos. Por otro lado, si excluimos el caso excepcional de Formentera, es en estas mismas Comunidades en donde el impacto desproporcional del bajo tamaño de los distritos produce efectos netamente bipartidistas en casi todos ellos, así: en 2 (Occidental y Oriental) de los 3 de Asturias, en 5 (Ávila, Palencia, Segovia, Soria y Zamora) de los 9 de Castilla y León, en 2 (Noroeste y Altiplano) de los 5 de Murcia, en 4 (todas las provincias menos Albacete) de los 5 de Castilla-La Mancha y en 1 (La Gomera) de los 7 de Canarias.

Si en comparación con el sistema general español el impacto desproporcional del tamaño de los distritos es menor, no sucede lo mismo con la desigualdad del voto, tal como hemos visto, ni con la base territorial o demográfica de los escaños, tal como se puede comprobar en la tabla 6. Así, si para las elecciones legislativas en España la distribución de escaños de base territorial suponen un 29 por 100 del total frente al 71 por 100 de los escaños de base demográfi$\mathrm{ca}$, en las elecciones autonómicas de las once Comunidades que no tienen distrito único ni son insulares el porcentaje de escaños de base territorial se duplica (52 por 100), superando ampliamente al de base demográfica (42 por 100) con todas sus limitaciones. De este modo, podemos clasificar estas Comunidades en tres grupos, así: el primero formado por las que superan significativamente dicho promedio (País Vasco, Comunidad Valenciana y Extremadura); un segundo grupo que se sitúa en torno al mismo, ya sea por arriba (Andalucía) o por abajo (Aragón, Murcia y Galicia); y un tercer grupo integrado por aquellas en las que la proporción de escaños de base demográfica es mayor (Asturias, Cataluña y Castilla y León). 


\section{TABLA 6}

Distribución de escaños de base territorial o demográfica en las CC.AA., 1993-1996*

\begin{tabular}{|c|c|c|c|}
\hline & $\begin{array}{c}(1) \\
\text { Escaños de } \\
\text { base territorial } \\
(\%)\end{array}$ & $\begin{array}{c}(2) \\
\text { Escaños de } \\
\text { base demográfica } \\
(\%)\end{array}$ & $\begin{array}{c}\text { Total } \\
\text { de escaños }\end{array}$ \\
\hline 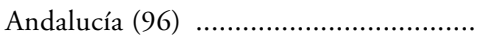 & $59 \quad(8)$ & 41 & 109 \\
\hline 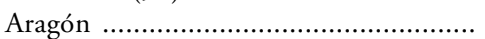 & $58(13)$ & 42 & 67 \\
\hline 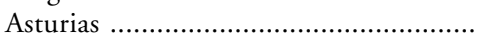 & $13(2)$ & 87 & 87 \\
\hline Castilla y León & $32(3)$ & 68 & 84 \\
\hline Castilla-La Mancha & $53 \quad(5)$ & 47 & 47 \\
\hline Cataluña $(95)^{* *}$ & 13 (6) & 87 & 135 \\
\hline Comunidad Valenciana .......................... & $67(20)$ & 33 & 89 \\
\hline Extremadura & $62(20)$ & 38 & 65 \\
\hline Galicia (93) & $53(10)$ & 47 & 75 \\
\hline Murcia & $56(5)$ & 44 & 45 \\
\hline 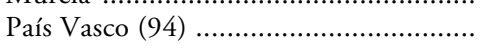 & $100(25)$ & 0 & 75 \\
\hline (2) & 58 & 42 & 836 \\
\hline
\end{tabular}

* Se han excluido las CC.AA. con distrito único: Cantabria, Madrid, Navarra y La Rioja. Igualmente, no se tienen en cuenta las insulares (Baleares y Canarias), cuyas leyes asignan un número fijo para cada isla.

** Cataluña asigna un número mínimo de 13 escaños para Girona, Tarragona y Lleida y luego aplica el criterio demográfico de forma diferencial para estas provincias (1 diputado por cada 40.000 habitantes) y para Barcelona (1 diputado por cada 50.000 habitantes).

(1) Proporción de escaños asignados según criterios territoriales. Entre paréntesis, el número mínimo de escaños por circunscripción o distrito.

(2) Proporción restante de escaños asignados con criterios demográficos.

FUENTE: Elaboración propia.

\section{BARRERAS MÍNIMAS DE VOTO Y UMBRALES DE INCLUSIÓN}

Un elemento adicional de la limitación del pluralismo político y, por tanto, de la productividad representativa del sistema electoral es la fijación de barreras mínimas de votos para acceder al reparto de escaños, que, si en el nivel nacional de las elecciones legislativas se ha mostrado innecesario por el impacto desproporcional de otros componentes del sistema electoral, sí tiene incidencia en el nivel autonómico. La variedad de barreras entre Comunidades Autónomas es evidente, si se observa la tabla 7. De esta tabla y según este criterio se pueden definir cuatro tipos o grupos de Comunidades Autónomas, así: el primero es el grupo mayoritario de siete (Andalucía, Aragón, Asturias, Castilla y León, Castilla-La Mancha, Cataluña y Navarra), que sigue la pauta nacional del 3 por 100 a nivel de distrito 
o circunscripción; el segundo grupo lo forman otras seis Comunidades (Baleares, Cantabria, Galicia, La Rioja, Madrid y el País Vasco), que elevan esa proporción hasta el 5 por 100 también en cada distrito; el tercer grupo lo integran Murcia y la Comunidad Valenciana, que elevan más el listón al aplicar ese mismo 5 por 100 al conjunto regional; finalmente, está en cuarto lugar los casos singulares de Canarias $^{2}$, que aplica alternativamente un 3 por 100 al nivel regional o un 20 por 100 a nivel de distrito, y Extremadura, que aplica, también indistintamente, un 5 por 100 a nivel de distrito o regional, siempre que en este segundo caso se hayan presentado candidaturas en las dos provincias.

\section{TABLA 7}

Barreras minimas de votos, candidaturas y votos sin representación y umbral de inclusión en las CC.AA., 1993-1996

\begin{tabular}{|c|c|c|c|c|c|}
\hline & $\begin{array}{l}\text { Barreras } \\
\text { (1) }\end{array}$ & $\begin{array}{l}N .^{\circ} \text { de } \\
\text { cand. sin } \\
\text { repres. } \\
(2)\end{array}$ & $\begin{array}{c}\text { Porcent. } \\
\quad \text { de } \\
\text { votos sin } \\
\text { repres. }\end{array}$ & $\begin{array}{c}\text { Umbral } \\
\text { de } \\
\text { inclusión } \\
\text { (3) }\end{array}$ & $\begin{array}{l}\text { Último } \\
\text { partido } \\
\text { (4) }\end{array}$ \\
\hline Andalucía (96) & $3 \mathrm{D}$ & $7 / 11$ & 0,87 & $6,69(6,9)$ & PA (CA) \\
\hline Aragón & $3 \mathrm{D}$ & $4 / 9$ & 0,71 & $4,86(5,6)$ & CHA (Z) \\
\hline Asturias & $3 \mathrm{D}$ & $6 / 10$ & 3,37 & $3,18(3,5)$ & PAS (Ce) \\
\hline Baleares & $5 \mathrm{D}$ & -17 & - & $0,32(49,4)$ & $\operatorname{AIPF}(\mathrm{Fo})$ \\
\hline Canarias & $3 \mathrm{R} / 20 \mathrm{D}$ & $11 / 16$ & 8,66 & $0,26(43,2)$ & AHI (Hi) \\
\hline Cantabria & $5 \mathrm{R} / \mathrm{D}$ & $4 / 9$ & 1,88 & 7,36 & IU \\
\hline Castilla y León . & $3 \mathrm{D}$ & $16 / 20$ & 4,09 & $2,55(12,8)$ & UPL (LE) \\
\hline Castilla-La Mancha ….......................... & $3 \mathrm{D}$ & $10 / 13$ & 1,35 & $7,6(10,3)$ & IU (AB) \\
\hline 95) .................. & $3 \mathrm{D}$ & $9 / 14$ & 0,91 & $9,7(6,4)$ & IC $(\mathrm{T})$ \\
\hline idad Valenciana & $5 \mathrm{R}$ & $10 / 14$ & 3,61 & $7,01(4,4)$ & UV (CS) \\
\hline$\ldots$ & $5 \mathrm{D}$ & $2 / 6$ & 1,34 & $3,81(5,9)$ & EU (CC) \\
\hline Galicia (93) & $5 \mathrm{D}^{*}$ & $9 / 12$ & 4,92 & $18,7(3,9)$ & BNG (C) \\
\hline (1) & $5 \mathrm{R} / \mathrm{D}$ & $13 / 16$ & 3,02 & 16 & IU \\
\hline Murcia & $5 \mathrm{R}$ & $6 / 9$ & 2,32 & $12,48(11,5)$ & ) IU (Ca) \\
\hline 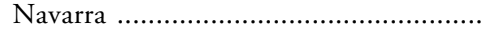 & $3 \mathrm{R} / \mathrm{D}$ & $4 / 10$ & 4,15 & 4,57 & EA \\
\hline 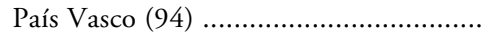 & $5 \mathrm{D}$ & $1 / 8$ & 0,14 & $2,68(18,5)$ & $\mathrm{UA}(\mathrm{Al})$ \\
\hline 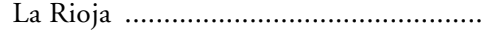 & $5 \mathrm{R} / \mathrm{D}$ & $1 / 5$ & 0,83 & 6,7 & PR \\
\hline
\end{tabular}

(1) Porcentaje a nivel de circunscripción (D) o regional (R).

(2) Número de candidaturas excluidas sobre el total de candidaturas presentadas.

(3) Porcentaje de votos obtenidos por el partido con menor número de escaños. Entre paréntesis, porcentaje más bajo que da derecho a escaño en uno de los distritos.

(4) Partido con menor número de escaños. Entre paréntesis, circunscripción en que obtiene el escaño con menor proporción de votos.

* Galicia elevó la barrera del 3 al 5 por 100 mediante una reforma electoral previa a las últimas elecciones de 1993.

FUENTE: Elaboración propia.

Con la reforma ya citada del Estatuto de Canarias se revisan al alza las barreras mínimas, elevándolas al 6 por 100 a nivel regional y al 30 por 100 de cada isla, asegurando la representación, en cualquier caso, a la lista más votada en una isla. 
Como ya se ha comprobado a nivel nacional, los efectos desproporcionales combinados del sistema de distritos y la fórmula de escrutinio son tan fuertes que han hecho innecesaria la aplicación de las barreras mínimas, salvo lo ocurrido al CDS en las legislativas de 1993; sin embargo, en el ámbito regional el mayor nivel de competencia abre las expectativas electorales de muchas opciones de implantación local, haciendo que sea elevado el número de candidaturas que concurren y que las barreras puedan jugar su papel efectivo. De hecho, como se puede comprobar, en el último período el número medio de candidaturas por Comunidad Autónoma es de 12, oscilando entre el mínimo de 5 de La Rioja y el máximo de 20 de Castilla y León. Si exceptuamos los casos de Baleares, el País Vasco y La Rioja, en los que no queda excluida ninguna o casi ninguna candidatura de las que se presentan, en el resto quedan sin representación o la mayoría de las que concurren (Castilla y León, Madrid, Comunidad Valenciana, Canarias, Galicia, Cataluña, Andalucía, Murcia y Asturias) o un número menor (Aragón, Cantabria, Navarra y Extremadura). Sin embargo, la proporción de votos que queda sin representación es mucho menor, no superando por término medio el 2,5 por 100 y oscilando entre 0 por 100 de las Baleares o el 0,14 por 100 del País Vasco y el 8,66 por 100 de las Canarias. Los casos más llamativos son los de IUC, con el 5,1 por 100 de los votos en Canarias (el 7,68 por 100 en La Gomera), y los de EU, con el 3,11 por 100 en Galicia (el 3,88 por 100 en Pontevedra y el 3,7 por 100 en La Coruña), siendo las únicas Comunidades donde el tercer partido de ámbito nacional (IU) no obtiene representación.

La aplicación de las barreras, con todo, ha resultado muy excepcional en las Comunidades Autónomas. Si tomamos en cuenta las últimas elecciones regionales, las barreras habrán sido de aplicación en sólo dos casos: para excluir a la UPV, con el 4,25 por 100 de los votos en Castellón (el 2,73 por 100 a nivel regional), y a BATZARRE en Navarra, con el 2,19 por 100 de los votos (como ya ocurriera con EE en la anterior legislatura). Excluidas las cuatro Comunidades con candidaturas representadas en un único distrito (Baleares, Canarias, Castilla y León y el País Vasco), el porcentaje medio de inclusión del partido con menos escaños en la circunscripción en que obtiene el escaño con menor proporción de votos es del 7,16 por 100, oscilando entre el mínimo del 3,5 por 100 del PAS en el distrito Centro de Asturias y el máximo del 16 por 100 de IU en Madrid. La excepción la suponen los casos de AIPF (con el 49,4 por 100 de los votos en Formentera), AHI (con el 43,2 por 100 en El Hierro), UPL (con el 12,8 por 100 en León) y UA (con el 18,5 por 100 en Álava), que definen los mínimos, sin embargo, a nivel de sus respectivas Comunidades Autónomas.

A pesar del pluralismo de partidos de las Comunidades Autónomas y de la mayor talla de sus distritos, los efectos desproporcionales del sistema, la tendencia creciente a la concentración del voto en dos grandes opciones y los conocidos efectos "psicológicos» (Shugart, 1985; Blais y Carty, 1991), que se vienen manifestando a través del «voto útil» (Gunther, 1989), han producido 
esta elevación de los niveles de inclusión, que es uno de los objetivos selectivos y estructurales de nuestro sistema electoral.

\section{DESPROPORCIONALIDAD ENTRE VOTOS Y ESCAÑOS}

Un elemento común al conjunto de las Comunidades Autónomas y que homogeneiza todo tipo de competición electoral en España, exceptuado el sistema mayoritario del Senado, es la fórmula de escrutinio de la variante D'Hondt de las fórmulas proporcionales de media más elevada, que, como ya se ha estudiado suficientemente, contribuye por sí misma a generar desproporcionalidad (Lijphart, 1986; Taagepera y Laakso, 1980). Sin embargo, el verdadero efecto corrector de esta fórmula tiene que ver más con el tamaño de la circunscripción, como han demostrado sobradamente Lijphart (1987: 178 s.), Gallagher (1991: 48) y Nohlen (1991: 312), entre otros.

El estudio de tales efectos para el caso español llega a la conclusión clara de que el sistema electoral español manifiesta las mayores dosis de desproporcionalidad de los países con sistemas proporcionales, situándose muy cerca de países, como Francia o el Reino Unido, con sistemas mayoritarios (Lijphart, 1985: 12 y 1987: 178 s.; Gallagher, 1991: 46; Montero, 1992; Montero, Llera y Torcal, 1992).

Después de nuestro estudio anterior hemos de suponer que tales efectos desproporcionales han de reducirse de forma sensible en el ámbito de las Comunidades Autónomas, debido precisamente a la mayor magnitud de sus circunscripciones. En la tabla 8 mostramos el cuadro final de pérdidas y ganancias de los partidos con representación parlamentaria en todas las Comunidades Autónomas en el último período 1993-1996, al comparar sus porcentajes de votos y escaños, deduciéndose con claridad que los efectos desproporcionales del sistema se reducen a la mitad en el ámbito autonómico. Se cumple la pauta general de que salen beneficiados los dos grandes partidos de cada distrito, que no siempre son los dos primeros de cada Comunidad, por el efecto combinado de una implantación diferencial y la magnitud de los distritos, así como el castigo de los pequeños y medianos con una distribución más o menos homogénea de sus apoyos electorales. Las Comunidades que presentan excepciones son Cantabria, Madrid, Navarra y el País Vasco. En el resto, el primer partido obtiene una ganancia media de alrededor de 4 puntos, que es menos de la mitad de la cosechada por el primer partido (UCD, PSOE y PP) en todas las elecciones legislativas en España (9,3); el segundo partido consigue unos 2 puntos, la mitad que el primero, reduciendo igualmente la diferencia media entre votos y escaños del segundo partido (PSOE y PP) en España entre 1977 y 1996 (4,1); finalmente, el tercer partido tiene una pérdida media de un 1,6 por 100 , que reduce también a la mitad los castigos de los terceros partidos españoles (AP, PCE/IU, UCD y CDS) en todas las elecciones legislativas cifrados en un promedio del 3,5 por 100. Se puede comprobar, además, cómo en 
aquellas Comunidades en las que existen magnitudes de distrito críticas se incrementa sensiblemente la desproporcionalidad media (las dos Castillas, Murcia, Asturias y Baleares). La fragmentación y la magnitud cántabra y navarra reparten las ganancias casi por igual, lo mismo que sucede en Madrid por la gran magnitud de su distrito único, mientras que en el País Vasco, además de la fragmentación, el gran efecto distorsionante lo causan el impacto localista de UA y la igualdad de magnitud entre los distritos, que priman o castigan la implantación diferencial.

\section{TABLA 8}

Desproporcionalidad votos/escaños de los partidos parlamentarios de las CC.AA., 1993-1996*

\begin{tabular}{|c|c|c|c|c|c|c|}
\hline & $P P$ & PSOE & $I U$ & $N N / R R .1$ & $N N / R R .2$ & $N N / R R .3$ \\
\hline Andalucía (96) & $+2,9$ & $+4,1$ & -2 & $-2,7(\mathrm{PA})$ & - & - \\
\hline 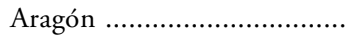 & $+2,4$ & $+2,4$ & $-2,2$ & $+0,5$ (PAR) & $-1,9$ (CHA) & - \\
\hline Asturias ............. & +5 & $+4,2$ & $-3,4$ & $-1,2$ (PAS) & - & - \\
\hline Baleares & $+6,2$ & +3 & $-1,6$ & $-2 \quad(\mathrm{PSM})$ & $-2,3(\mathrm{UM})$ & $+1,0(\mathrm{Ot})$ \\
\hline Canarias & -1 & +4 & - & $+4,2(\mathrm{CC})$ & $+2 \quad(\mathrm{PCN})$ & $+1,7$ (AHI) \\
\hline Cantabria ... & $+0,5$ & $+0,9$ & $+0,6$ & +1,4 (UPCA) & +0,4 (PRC) & \\
\hline Castilla y León & $+7,7$ & $+2,4$ & $-3,6$ & $-0,5$ (UPL) & - & - \\
\hline Castilla-La Mancha & $+2,7$ & $+5,3$ & $-5,6$ & - & - & - \\
\hline Cataluñ & $-0,1$ & $+0,2$ & $-1,7$ & $+3 \quad(\mathrm{CiU})$ & $+0,5$ (ERC) & - \\
\hline dad Valenciana ..... & $+4,1$ & $+2,1$ & $-0,5$ & $-1 \quad(\mathrm{UV})$ & - & - \\
\hline Extremadura & $+2,5$ & $+4,1$ & $-1,6$ & $-1,8(\mathrm{EU})$ & - & - \\
\hline Galicia (93) ... & $+4,8$ & $+1,5$ & - & $-1,7$ (BNG) & - & - \\
\hline 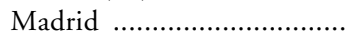 & $+1,1$ & $+1,3$ & +1 & - & - & - \\
\hline Murcia …............................... & $+5,6$ & $+1,3$ & $-3,5$ & - & - & - \\
\hline 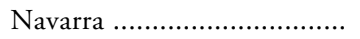 & - & $+1,1$ & $+0,6$ & +2,6 (UPN) & $+1,4(\mathrm{CDN})$ & $+0,8(\mathrm{HB})$ \\
\hline País Vasco (94) ...................... & $+0,8$ & $-0,8$ & -1 & $-0,3$ (PNV) & $-1 \quad(\mathrm{HB})$ & $+4,3(\mathrm{UA})$ \\
\hline 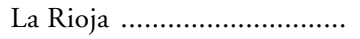 & $+2,6$ & $+1,9$ & $-1,2$ & $-0,7(\mathrm{PR})$ & - & - \\
\hline
\end{tabular}

* Diferencias entre los porcentajes de votos y escaños. En Baleares, además, salen beneficiadas las candidaturas insulares de EV (en Ibiza) y AIPF (en Formentera). En Navarra también sale perjudicada EA con una diferencia de -0,6. En el País Vasco, por el contrario, EA sale beneficiada con un 0,9 por 100 .

FUENTE: Elaboración propia.

Otra forma de evaluar las limitaciones a la proporcionalidad de los sistemas electorales de las Comunidades Autónomas es mediante el recurso, por un lado, a indicadores sintéticos de proporcionalidad y, por otro lado, a la comparación de esos mismos indicadores con los correspondientes a nivel nacional o internacional. En la tabla 9 mostramos tres de estos indicadores sintéticos de la 
desproporcionalidad aplicados al ámbito parlamentario. En primer lugar, el indice de fragmentación (parlamentaria) de Rae (1971), que para el conjunto de los parlamentos autonómicos actuales da un promedio moderado de 0,64 , no muy distinto de la media de 0,61 que resulta para el Congreso de los Diputados entre 1977 y 1996 (entre el mínimo de 0,57 de 1982 y el máximo de 0,64 de 1989), pero con un recorrido que oscila entre el mínimo del 0,52 de Castilla-La Mancha y el máximo del 0,82 del País Vasco. Esto nos permite clasificar a las Comunidades Autónomas en tres grupos casi iguales, así: el primero de las seis menos fragmentadas, formado por Castilla-La Mancha, Castilla y León, Murcia, Extremadura, Galicia y La Rioja, todas ellas por debajo de 0,64, con tres o cuatro partidos parlamentarios y con mayorías absolutas o amplias; el grupo intermedio, cuya fragmentación se sitúa entre 0,6 y 0,7 , formado por Madrid, Andalucía, Asturias, Comunidad Valenciana y Baleares, con mayorías más frágiles, mayor número de partidos o terceros partidos importantes; y el tercer grupo es el de las más fragmentadas, con índices por encima del 0,7 y que lo forman el País Vasco, Navarra, Cantabria, Cataluña, Aragón y Canarias, caracterizados por un mayor pluralismo de partidos y la ausencia de mayorías sólidas.

El indice de desproporcionalidad de Lijphart (1987: 178s.), que mejora el anterior al neutralizar el efecto distorsionante de los pequeños partidos, alcanza un promedio de 4,9 para el conjunto de las Comunidades Autónomas, muy inferior al 6,7 que resulta para todas las elecciones al Congreso de los Diputados, lo que nos vuelve a dar idea de cómo se rebaja la desproporcionalidad a nivel autonómico para colocarse en el umbral mínimo de los sistemas nacionales con la misma fórmula de escrutinio, de acuerdo con la estimación de Lijphart (1988). Sin embargo, este índice sigue estando muy por encima del 2,4 por 100 medio calculado por Lijphart (1987: 174 s.) para los países con sistemas proporcionales. De la comparación de las distintas Comunidades resulta, de nuevo, una tipología de tres grupos, así: el primer grupo de las seis menos desproporcionales con un índice por debajo del 4 (Madrid, Cantabria, Cataluña, La Rioja, Navarra y la Comunidad Valenciana); un segundo grupo de otras seis con una desproporcionalidad media, entre 4 y 6 , formado por Galicia, el País Vasco, Aragón, Extremadura, Murcia y Andalucía; finalmente, el grupo más desproporcional que se sitúa por encima del umbral 6 , superior al estimado por Lijphart para los sistemas de fórmula D'Hondt, y que lo encabeza Baleares con 11, seguida de Castilla y León, Asturias, Castilla-La Mancha y Canarias, en las que se deja sentir con fuerza el efecto de la menor magnitud de los distritos electorales.

Finalmente, el indice del número de partidos (Laakso y Taagepera, 1979; Taagepera y Shugart, 1989), en sus versiones electoral y parlamentaria, permite comparar cuántos partidos compiten electoralmente y cuántos existen parlamentariamente, teniendo en cuenta en ambos casos sus tamaños relativos y dando, por tanto, mayor información sobre la medida más aproximada de la desproporcionalidad de conjunto. Los promedios del número efectivo de parti- 


\section{TABLA 9}

Fragmentación parlamentaria, número efectivo de partidos e indice de desproporcionalidad en las elecciones autonómicas, 1993-1996*

\begin{tabular}{|c|c|c|c|c|c|}
\hline & $\begin{array}{c}F p \\
\text { Fragment. } \\
\text { parlament. } \\
\text { (1) }\end{array}$ & $\begin{array}{c}D \\
\text { Indice } \\
\text { desproporc. } \\
(2)\end{array}$ & $\begin{array}{c}\text { Nep } \\
\text { Num. efect. } \\
\text { Pp. parlam. } \\
\text { (3) }\end{array}$ & $\begin{array}{c}\text { Nee } \\
\text { Núm. efect. } \\
\text { Pp. elect. } \\
\text { (3) }\end{array}$ & $\begin{array}{c}\text { Difer. } \\
\text { Nee-Nep } \\
\text { (4) }\end{array}$ \\
\hline Andalucía (96) ......... & 0,62 & $5,6(+)$ & 2,6 & 2,9 & 0,3 \\
\hline Aragón ........................ & 0,71 & $4,7(+)$ & $3,4(+)$ & 3,7 & 0,3 \\
\hline Asturias ...................... & $0,62(-)$ & $6,9(+)$ & 2,6 & 3 & 0,4 \\
\hline 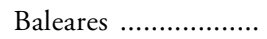 & 0,65 & $11,0(+)$ & 2,9 & 4,8 & 1,9 \\
\hline Canarias ...................... & $0,70(-)$ & $6,4(+)$ & $3,3(-)$ & 3,7 & 0,4 \\
\hline Cantabria .................... & 0,76 & $1,9(-)$ & $4,2(+)$ & 4,3 & 0,1 \\
\hline Castilla y León ......... & $0,53(-)$ & $7,1(+)$ & 2,1 & 2,7 & 0,6 \\
\hline Castilla-La Mancha . & $0,52(-)$ & $6,8(+)$ & 2,1 & 2,4 & 0,3 \\
\hline Cataluña (95) ........... & 0,71 & $2,7(-)$ & 3,4 & 3,7 & 0,3 \\
\hline Com. Valenciana .... & $0,63(-)$ & $3,8(-)$ & 2,7 & 3,2 & 0,5 \\
\hline Extremadura ............... & 0,58 & $5,0(+)$ & 2,4 & 2,8 & 0,4 \\
\hline Galicia (93) .............. & $0,59(-)$ & $4,0(-)$ & 2,4 & 2,8 & 0,4 \\
\hline Madrid ....................... & $0,60(-)$ & $1,7(-)$ & 2,5 & 2,9 & 0,4 \\
\hline Murcia ...................... & $0,54(-)$ & $5,2(-)$ & 2,2 & 3,3 & 1,1 \\
\hline Navarra ....................... & 0,76 & $3,5(-)$ & $4,2(+)$ & 5 & 0,8 \\
\hline País Vasco (94) ........ & 0,82 & $4,5(+)$ & 5,6 & 5,6 & 0 \\
\hline La Rioja .................... & 0,59 & $3,2(-)$ & 2,4 & 2,6 & 0,2 \\
\hline
\end{tabular}

* Los ( \pm ) indican la evolución de los distintos índices respecto al período anterior 1989-1992.(1) Índice de fragmentación parlamentaria de Rae (1971).

(2) Índice de Desproporcionalidad de Lijphart (1987).

(3) Índice de Número Efectivo de Partidos parlamentarios (Nep) o electorales (Nee) de Laakso y Taagepera (1979).

(4) La diferencia entre Nee y Nep.

FUENTE: Elaboración propia.

dos electorales y parlamentarios de 3,5 y 3, respectivamente, para el conjunto de las Comunidades Autónomas se diferencian ligeramente de la media obtenido para el Congreso de los Diputados, aunque no cambian nuestra posición en el ránking de los países estimado por Montero (1992). El cambio significativo es la reducción drástica de las diferencias entre los campos electoral y parlamentario, que pasa del 1,21 en el ámbito legislativo, el más elevado de Europa, al 0,5 situado por debajo de la media europea. Tomando como referencia tales diferencias, que son las que nos dan la medida del efecto reductor del sistema electoral, podemos clasificar a las Comunidades en dos grandes grupos, según se sitúen por encima o por debajo de tal promedio, así: el de las más desproporcionales está formado por Baleares, Murcia, Navarra y Castilla y León, 
mientras que el orden de las menos desproporcionales lo encabeza el País Vasco, seguido por Cantabria, La Rioja, Cataluña, Aragón, Andalucía, Castilla-La Mancha, Canarias, Asturias, Madrid, Galicia, Extremadura y la Comunidad Valenciana.

\section{LOS SISTEMAS DE PARTIDOS Y SU FORMATO}

Cuando se diseña en 1977 el sistema electoral español, lo que se buscaba era garantizar la mayoría absoluta de escaños a la UCD a partir del 37 por 100 de los votos, favoreciendo para ello a las zonas rurales y tradicionales frente a las urbanas e industriales con un adecuado sistema de distritos; además, se trataba de potenciar grandes opciones políticas reduciendo en lo posible la inicial fragmentación electoral y sin excluir a los principales partidos nacionalistas de Euskadi y Cataluña. Por consiguiente, el rendimiento político obtenido por el sistema electoral ha sido la conformación de un sistema de partidos que, garantizando un pluralismo moderado, ha facilitado la producción alternativa de gobiernos estables y no ha excluido a fuerzas políticamente relevantes (AP/CP, PCE/IU, PNV y CiU, sobre todo).

Después de casi veinte años, el sistema sigue intacto e institucionalizado, produciendo efectos claramente mayoritarios para los principales partidos, que se combinan con el mantenimiento de la representación, cada vez más relevante políticamente, de los partidos nacionalistas y algunos regionalistas, incluso llegando a producir mayorías absolutas «manufacturadas» (Rae, 1977; Blais y Carty, 1987) entre 1982 y 1989.

En lo fundamental, este sistema a nivel autonómico tiene un rendimiento político muy similar. Un rápido vistazo a la tabla 10 , en la que mostramos los sistemas de partidos parlamentarios de las 17 Comunidades Autónomas, hace bastante perceptible el paralelismo con la dinámica nacional, aunque no sea más que porque en 14 de ellas los actores principales de la escena política son los dos protagonistas de la arena nacional. En las tres restantes (Cataluña, Euskadi y Canarias) el protagonismo lo comparten con los partidos nacionalistas moderados, que juegan un papel relevante en la gobernabilidad del Estado. En segundo plano se sitúa el tercer partido nacional (IU), presente en todas las Comunidades, a excepción de Canarias y Galicia. Finalmente, en casi todas ellas también (menos en Madrid, Murcia y Castilla-La Mancha) hay sitio para otros 22 partidos nacionalistas, regionalistas o simplemente territoriales. 
TABLA 10

Distribución de escaños en los Parlamentos autonómicos, 1993-1996

\begin{tabular}{|c|c|c|c|c|c|c|}
\hline & $P P$ & PSOE & $I U$ & & $N N / R R$. & Total \\
\hline Andalucía (96) & 40 & 52 & 13 & 4 & $(\mathrm{PA})$ & 109 \\
\hline 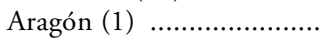 & 27 & 19 & 5 & 16 & (PAR, CHA) & 67 \\
\hline Asturias ................................. & 21 & 17 & 6 & 1 & (PAS) & 45 \\
\hline Baleares .............................. & 30 & 16 & 3 & 10 & (PSM, UM, EU, AIPF) & 59 \\
\hline 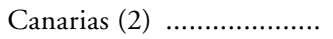 & 18 & 16 & - & 26 & (CC, PCN, AHI) & 60 \\
\hline Cantabria (3) ...................... & 13 & 10 & 3 & 13 & (UPCA, PRC) & 39 \\
\hline Castilla y León ................. & 50 & 27 & 5 & 2 & (UPL) & 84 \\
\hline Castilla-La Mancha .......... & 22 & 24 & 1 & - & & 47 \\
\hline Cataluña (95) (4) .............. & 17 & 34 & 11 & 73 & $(\mathrm{CiU}, \mathrm{ERC})$ & 135 \\
\hline Comunidad Valenciana ... & 42 & 32 & 10 & 5 & (UV) & 89 \\
\hline Extremadura ...................... & 27 & 31 & 6 & 1 & $(\mathrm{EU})$ & 65 \\
\hline Galicia $(93)$...................... & 43 & 19 & - & 13 & $(\mathrm{BNG})$ & 75 \\
\hline 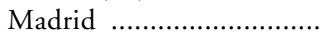 & 54 & 32 & 17 & - & & 103 \\
\hline Murcia ............................... & 26 & 15 & 4 & - & & 45 \\
\hline Navarra (5) & - & 11 & 5 & 34 & (UPN, CDN, HB, EA) & 50 \\
\hline País Vasco (94) (6) .......... & 11 & 12 & 6 & 46 & $(\mathrm{PNV}, \mathrm{HB}, \mathrm{EA}, \mathrm{UA})$ & 75 \\
\hline La Rioja ............................... & 17 & 12 & 2 & 2 & (PR) & 33 \\
\hline
\end{tabular}

FUENTE: Elaboración propia.

(1) El PAR es el tercer partido, con 14 escaños.

(2) CC es el primer partido, con 21 escaños; PCN, 4; AHI, 1.

(3) La UPCA del ex presidente Hormaechea es el tercer partido, con 7 escaños.

(4) $\mathrm{CiU}$ es el primer partido, con 60 escaños.

(5) UPN es el primer partido, con 17 escaños; CDN del ex presidente Alli es el tercero, con 10 escaños; HB obtiene 5, y EA, 2.

(6) El PNV es el primer partido, con 22 escaños; HB, 11; EA, 8, y UA, 5.

Esta estructura bipolar de la competencia electoral se puede ver en la siguiente tabla 11. PP y PSOE ocupan las primeras posiciones en 13 Comunidades, sumando entre el 58 y el 90 por 100 de los votos de Cantabria y Castilla-La Mancha, respectivamente, y habiendo incrementado tal concentración de voto en 9 de ellas en el último período. En Canarias el PP acompaña a CC en la segunda posición, mientras que en las otras tres (Cataluña, País Vasco y Navarra) lo hace el PSOE con CiU, PNV y UPN, respectivamente, si bien en todas ellas con niveles de concentración de voto mucho menores ( $\mathrm{y}$, en general, decrecientes) y que oscilan entre el mínimo del 46 por 100 del País Vasco y el máximo del 66 por 100 de Cataluña. Finalmente, otro dato digno de tener en cuenta es el indice de competitividad, que resulta de calcular la distancia entre los porcentajes de votos del primero y segundo partidos (da una media del 13 por 100 para el conjunto de las Comunidades Autónomas, que es mayor que este mismo parámetro a nivel legislativo nacional, oscilando entre el 1 por 100 de Castilla-La Mancha y el 29 por 100 de Galicia) y resultando mayoritariamente creciente (9), sobre todo, en las que gana el PP (y UPN). 
TABLA 11

Estructura de la competencia electoral en las CC.AA., 1993-1996

\begin{tabular}{|c|c|c|c|c|c|c|}
\hline \multirow{2}{*}{$\begin{array}{c}\begin{array}{c}\text { Dos partidos } \\
\text { princip. }\end{array} \\
\text { PP-PSOE }\end{array}$} & \multirow[t]{2}{*}{ CC.AA. } & \multirow[t]{2}{*}{$\begin{array}{c}\% \text { votos dos } \\
\text { primeros }\end{array}$} & \multirow[t]{2}{*}{$\begin{array}{l}\text { Evoluc. } \\
\text { concent. } \\
\text { electoral } \\
\quad(1)\end{array}$} & \multicolumn{2}{|c|}{$\begin{array}{l}\text { Partido } \\
\text { ganador } \\
\text { (ventaja } \% \\
\text { s/segundo) }\end{array}$} & $\begin{array}{l}\text { Evoluc. } \\
\text { ventaja } \\
\quad(1)\end{array}$ \\
\hline & & & & & & \\
\hline \multirow[t]{10}{*}{ a) $\mathrm{PP}$} & Aragón* & 63 & $=$ & PP & $(+12)$ & - \\
\hline & Asturias* & 76 & + & PP & $(+8)$ & - \\
\hline & Baleares & 69 & - & PP & $(+21)$ & + \\
\hline & Cantabria & 58 & + & PP & $(+7)$ & + \\
\hline & Castilla y León & 82 & + & PP & $(+23)$ & + \\
\hline & Comunidad Valenciana* & 77 & + & PP & $(+9)$ & - \\
\hline & Galicia (93) & 76 & $=$ & PP & $(+29)$ & + \\
\hline & Madrid & 81 & + & PP & $(+21)$ & + \\
\hline & Murcia* & 84 & + & PP & $(+21)$ & + \\
\hline & La Rioja* & 83 & $=$ & PP & $(+15)$ & + \\
\hline \multirow[t]{3}{*}{ b) $\mathrm{PSOE}$} & Andalucía (96) & 78 & + & PSOE & $(+10)$ & - \\
\hline & Castilla-La Mancha & 90 & + & PSOE & $(+1)$ & - \\
\hline & Extremadura & 83 & + & PSOE & $(+4)$ & - \\
\hline$C C-P P$ & Canarias* & 64 & + & $\mathrm{CC}$ & $(+2)$ & - \\
\hline$C i U-P S C$ & Cataluña (95) & 66 & - & $\mathrm{CiU}$ & $(+16)$ & - \\
\hline$U P N-P S O E$ & Navarra & 52 & - & UPN & $(+10)$ & + \\
\hline PNV-PSOE & País Vasco (94) & 46 & - & PNV & $(+12)$ & + \\
\hline
\end{tabular}

(1) Evolución en relación al período 1989-1992.

* Comunidades en las que se ha producido un cambio de mayoría en el último proceso electoral.

FUENTE: Elaboración propia.

Un efecto importante de nuestro sistema electoral sobre la estructura de la competencia electoral y sobre el sistema de partidos es la dinámica y el grado de concentración del voto en los dos principales partidos. El primer dato que es digno de tomarse en consideración es que la concentración media de voto en todas las elecciones autonómicas ( 71,7 por 100) es ligeramente superior a la observada en todas las elecciones legislativas $(69,8$ por 100$)$. En la tabla 12 podemos estudiar este fenómeno para cada una de las Comunidades Autónomas para todas las elecciones autonómicas y para las legislativas habidas en el último decenio, pudiendo comprobarse que se ha invertido la tendencia general, si tenemos en cuenta que, mientras que en las últimas elecciones autonómicas la concentración media se sitúa en el 72 por 100, a pesar de haber mantenido su ligera tendencia creciente, en las legislativas de 1996 ha superado el 76 por 100, siguiendo una tendencia de crecimiento sostenido desde 1977. Este diferencial, con mayor o menor intensidad, se produce prácticamente en todas las Comunidades Autónomas, con las únicas excepciones de Castilla-La Mancha, Cataluña, Madrid y el 


\section{TABLA 12}

Concentración del voto en las CC.AA. en elecciones autonómicas y generales, 1980-1996*

\begin{tabular}{|c|c|c|c|c|}
\hline \multirow[b]{2}{*}{ Andalucía } & \multicolumn{2}{|c|}{$\begin{array}{c}\text { Promedio autonómicas } \\
1980-1996\end{array}$} & \multicolumn{2}{|c|}{$\begin{array}{c}\text { Promedio generales } \\
1986-1996\end{array}$} \\
\hline & 73,7 & $(+)$ & 79,1 & $(+)$ \\
\hline Aragón & 64,2 & $(-)$ & 74,7 & $(+)$ \\
\hline 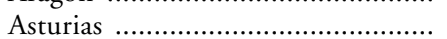 & 73,7 & $(+)$ & 76,1 & $(+)$ \\
\hline 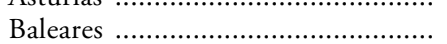 & 69,9 & $(-)$ & 78,4 & $(+)$ \\
\hline Canarias & 60,5 & $(+)$ & 62,7 & $(+)$ \\
\hline 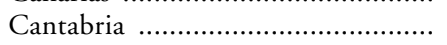 & 64,2 & $(-)$ & 81,0 & $(+)$ \\
\hline Castilla y León & 79,0 & $(+)$ & 82,2 & $(+)$ \\
\hline 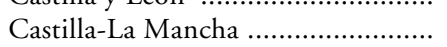 & 87,4 & $(+)$ & 86,6 & $(+)$ \\
\hline 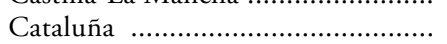 & 70,5 & $(-)$ & 68,9 & \\
\hline Comunidad Valenciana .................. & 74,4 & $(+)$ & 78,1 & $(+)$ \\
\hline 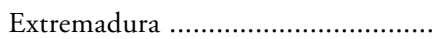 & 80,9 & $(+)$ & 85,5 & $(+)$ \\
\hline 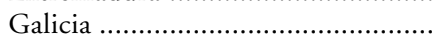 & 71,8 & $(+)$ & 78,9 & $(+)$ \\
\hline 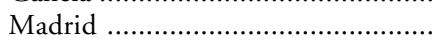 & 79,1 & $(+)$ & 76,5 & $(+)$ \\
\hline Murcia & 81,6 & $(+)$ & 84,4 & $(+)$ \\
\hline Navarra & 55,7 & $(-)$ & 66,6 & $(+)$ \\
\hline 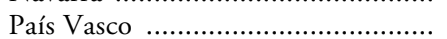 & 49,3 & $(-)$ & 48,4 & \\
\hline 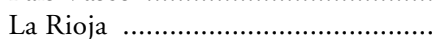 & 82,3 & $(+)$ & 83,8 & $(+)$ \\
\hline
\end{tabular}

* La concentración media española en todas las elecciones legislativas es del 69,83 por 100 y el recorrido va del mínimo del 63,76 por 100 en 1977 al 76,33 por 100 máximo en 1996.

$( \pm)$ Los signos indican la tendencia media a incrementarse o disminuir la concentración de voto en el período.

FUENTE: Elaboración propia.

País Vasco. Como ya señalábamos más arriba en relación con las últimas elecciones autonómicas, el recorrido de este parámetro para el conjunto de Comunidades desde 1980 va de la concentración media mínima del País Vasco (49,3 por 100) al máximo de Castilla-La Mancha (87,4 por 100), pudiendo clasificarlas en tres grupos, así: el primero de máxima concentración, superior al 79 por 100, formado por Castilla-La Mancha, La Rioja, Murcia, Extremadura, Madrid y Castilla y León; el segundo, de una concentración media que oscila entre el 65 y el 75 por 100, integrado por la Comunidad Valenciana, Andalucía, Asturias, Galicia, Cataluña y Baleares; y el tercero, por debajo del 65 por 100 medio de concentración, lo forman las cinco restantes (Aragón, Cantabria, Canarias, Navarra y el País Vasco), que están, a su vez, entre las más fragmentadas y plurales.

Como ya indicábamos, el principal efecto de los sistemas electorales se produce en relación con los formatos adquiridos por los sistemas de partidos parlamentarios, que son los que, finalmente, producen gobierno. Como puede comprobarse en la tabla 13, el mapa autonómico resultante abarca una impor- 
TABLA 13

Formato de los sistemas de partidos parlamentarios de las CC.AA., 1993-1996

\begin{tabular}{|c|c|c|c|c|c|c|c|}
\hline \multirow[b]{2}{*}{ Andalucía (96) } & $\begin{array}{l}\text { Núm. } \\
\text { de } \\
\text { partidos } \\
\text { parlam. }\end{array}$ & \multicolumn{2}{|c|}{$\begin{array}{l}\text { Primer } \\
\text { partido } \\
\text { (1) }\end{array}$} & \multirow{2}{*}{$\begin{array}{l}\text { Concent. } \\
\text { esc. } \\
1 .^{\circ} \text { y } 2 .^{\circ} \\
(2)\end{array}$} & \multicolumn{2}{|c|}{$\begin{array}{c}\text { Núm. } \\
\text { partidos } \\
\text { nac. y reg. } \\
\text { (3) }\end{array}$} & \multirow{2}{*}{$\begin{array}{c}\begin{array}{c}\text { Composición } \\
\text { Gobierno } \\
(4)\end{array} \\
\text { PSOE-PA }\end{array}$} \\
\hline & 4 & PSOE & $(54)$ & & 1 & (3) & \\
\hline Aragón ............. & 5 & PP & $(40)$ & 69 & 2 & $(24)$ & PP-PAR \\
\hline 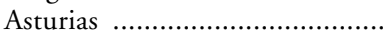 & 4 & PP & $(47)$ & 84 & 1 & (2) & $\mathrm{PP}^{*}$ \\
\hline Baleares & 7 & PP & $(51)$ & 78 & 4 & (17) & $\mathrm{PP}$ \\
\hline Canarias & 5 & $\mathrm{CC}$ & (35) & 66 & 3 & $(43)$ & CC-PP \\
\hline 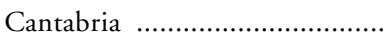 & 5 & PP & (33) & 59 & 2 & (33) & PP-PRC \\
\hline Castilla y León .......................... & 4 & $\mathrm{PP}$ & $(60)$ & 92 & 1 & (2) & PP \\
\hline Castilla-La Mancha ................... & 3 & PSOE & $(51)$ & 98 & - & & PSOE \\
\hline 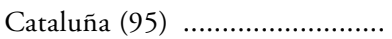 & 5 & $\mathrm{CiU}$ & $(44)$ & 70 & 2 & $(54)$ & $\mathrm{CiU}^{*}$ \\
\hline Comunidad Valenciana ............. & 4 & $\mathrm{PP}$ & $(47)$ & 83 & 1 & (6) & PP-UV \\
\hline Extremadura . & 4 & PSOE & $(48)$ & 89 & 1 & (2) & PSOE* \\
\hline 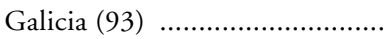 & 3 & PP & $(57)$ & 83 & 1 & (17) & $\mathrm{PP}$ \\
\hline 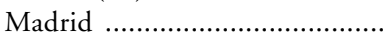 & 3 & PP & $(52)$ & 83 & - & & PP \\
\hline 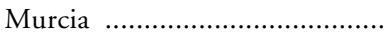 & 3 & PP & $(58)$ & 91 & - & & $\mathrm{PP}$ \\
\hline 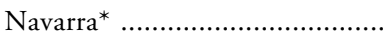 & 6 & UPN & (34) & 56 & 4 & $(68)$ & $\mathrm{UPN}^{*}$ \\
\hline País Vasco (94) & 7 & PNV & (29) & 45 & 4 & (61) & PNV-PSE/EE-EA \\
\hline 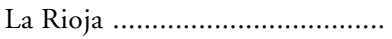 & 4 & PP & $(52)$ & 88 & 1 & (6) & PP \\
\hline
\end{tabular}

(1) Primer partido parlamentario (y porcentaje de escaños).

(2) Porcentaje de escańos acumulado por los dos partidos mayores.

(3) Número de partidos nacionalistas y regionalistas (y porcentaje de escaños).

(4) Composición de los gobiernos autónomos: ( ) monocolores con mayoría absoluta; (-) de coalición; $\left({ }^{*}\right)$ monocolores en minoría.

* Navarra ha sido gobernada hasta el verano de 1996 por la coalición PSN-CDN-EA, con apoyo parlamentario de IU.

FUENTE: Elaboración propia.

tante variedad de sistemas o subsistemas regionales de partidos. El efecto más generalizado ha significado la conformación de una tendencia mayoritaria que oscila entre el bipartidismo imperfecto (Duverger, 1974: 239 ss.; Balli, 1966) en once Comunidades y el pluralismo moderado (Sartori, 1980: 219 ss.) en otras cuatro. Podemos definir cinco tipos de subsistemas de partidos parlamentarios, así: el primer tipo, de bipartidismo imperfecto de tres partidos, o «dos partidos y medio", como dijera de Alemania, por primera vez, C. J. Friedrich (1946: 414), lo componen los tres partidos del sistema estatal en Castilla-La Mancha, Madrid y Murcia, por un lado, y los dos mayores partidos nacionales con otro nacionalista (BNG) en Galicia, por otro lado, todas ellas con gobierno monocolor con mayoría absoluta (en todas del PP, excepto el PSOE en la primera); el segundo tipo, también de bipartidismo imperfecto, aunque sea de 
cuatro o más partidos, lo componen los tres partidos nacionales más uno o varios partidos minoritarios de carácter regionalista en Andalucía, Asturias, Baleares, Castilla y León, Comunidad Valenciana, Extremadura y La Rioja, tres de ellas con gobiernos monocolores con mayoría absoluta del PP (Castilla y León y La Rioja), otras dos con gobiernos de coalición PSOE-PA (Andalucía) y PP-UV (Comunidad Valenciana) y las otras dos con gobiernos monocolores en minoría del PP (Asturias) y el PSOE (Extremadura); el tercer tipo, de pluralismo moderado de cinco partidos, lo componen también los tres partidos nacionales más dos partidos minoritarios de carácter regionalista con gobiernos de coalición entre el PP y el PAR (en Aragón) o el PRC (en Cantabria); el cuarto tipo, también de pluralismo moderado de cinco partidos, lo definen Canarias y Cataluña con presencia de los tres partidos nacionales, pero encabezados por un partido nacionalista a los que se añade otro minoritario y con gobiernos de coalición CC-PP (Canarias) o monocolor en minoría de CiU (Cataluña); finalmente, el quinto tipo es el de pluralismo extremo y polarizado (Sartori, 1980: 165 ss.) de 6 y 7 partidos de Navarra y el País Vasco, respectivamente, con los tres partidos nacionales más los tres nacionalistas vascos, a los que se añade el alavesista UA en el País Vasco, encabezados por el regionalista UPN (PP) en Navarra y el nacionalista PNV en el País Vasco, con gobierno monocolor minoritario de UPN en Navarra (tras la quiebra de la coalición PSOE-CDN-EA en 1996) y de coalición PNV-PSE-EA en el País Vasco.

\section{CONCLUSIONES}

Lo primero que cabe afirmar es la clara institucionalización de los sistemas electorales de las Comunidades Autónomas, que refuerzan el enraizamiento del sistema electoral español en su conjunto, precisamente por la continuidad y mimetismo de los rasgos básicos en ambos niveles políticos. A pesar de la tendencia armonizadora y de la homogeneidad mayoritaria de su funcionamiento, la heterogeneidad de sus efectos en algunas Comunidades Autónomas tiene que ver, sobre todo, con las especiales características de la territorialidad y la vida política de las mismas.

En general, al igual que para el conjunto de España, los sistemas electorales de las Comunidades Autónomas también han tenido efectos concentrados sobre los sistemas de partidos, aunque ligeramente más atenuados, contribuyendo a reducir la fragmentación parlamentaria y a facilitar la formación de gobiernos basados en mayorías homogéneas. Con todo, los sistemas autonómicos reducen de forma sensible la acusada desproporcionalidad del sistema español.

A la vista de la homogeneidad de la fórmula de escrutinio y de la escasa incidencia de las barreras mínimas, el sistema de distritos sigue siendo también aquí el principal factor crítico de la desigualdad del voto y de la desproporcionalidad estructural del sistema, así como el elemento diferencial entre Comu- 
nidades. Como prueba, baste el siguiente ejercicio que consiste en neutralizar sucesivamente los efectos de los sistemas de distritos y de la fórmula de escrutinio, así: si, en primer lugar, simulamos distritos únicos para las 13 Comunidades que no los tienen, el resultado es un intercambio medio de escaños del 4 por 100 , sobre todo a costa de los partidos mayoritarios y que, además, permitiría, de no aplicarse barreras de votos, el acceso de partidos en la Comunidad Valenciana (UPV, con 2 escaños) y Galicia (EU, con 2 escaños); en segundo lugar, si mantenemos igualmente neutralizado el efecto distorsionante de los distritos y cambiamos, al mismo tiempo, la forma de escrutinio hacia un sistema proporcional puro de resto mayor, el intercambio medio adicional de escaños de las 17 Comunidades por este factor no superaría el 2 por 100, permitiendo, sin embargo, el acceso al reparto de escaños de una docena de nuevos partidos en Asturias (CAS-CDS), Canarias (CGC y CDS-UC), Castilla y León (TC-PNC, SI, AIAV y PB), Madrid (LV-GU y LVA), Navarra (Batzarre y NA) y Cataluña (AEC). Esta doble combinación sobre los sistemas de partidos y la gobernabilidad tendría el efecto de debilitar la mayoría de gobierno en Aragón, Canarias y Comunidad Valenciana, complicaría la gobernabilidad en Asturias, Extremadura, Navarra y Cataluña, dejaría en minoría al PP en Baleares y al PSOE en Castilla-La Mancha y no cambiaría nada de forma significativa en las ocho restantes.

La continuidad normativa de los sistemas electorales autonómicos con respecto al nacional y su relativa homogeneidad interterritorial no han impedido, sin embargo, la producción de una considerable variedad de sistemas de partidos, aunque con un predominio claro y creciente del formato del bipartidismo imperfecto en once Comunidades y, en menor medida, del pluralismo moderado en otras cuatro, que conviven con la excepcionalidad del pluralismo extremo o polarizado del País Vasco y Navarra. Ahora bien, el mayor pluralismo de los sistemas de partidos autonómicos, facilitados por sus sistemas electorales, sin mermar significativamente ni su bipolarización ni la gobernabilidad, ha tenido, al menos, dos efectos positivos: el primero, dar acceso a la escena parlamentaria de nuevos actores políticos locales; y el segundo, y más importante, ensayar las fórmulas de gobiernos de coalición en cada vez mayor número de Comunidades.

De ahí que las voces de reforma o de mayor armonización formal de los sistemas electorales autonómicos, que últimamente venimos escuchando, tengan poco que ver con la institucionalización y los rendimientos alcanzados por los mismos. 


\section{REFERENCIAS}

BALLI, Giovanni (1966): Il bipartitismo imperfecto, Milán: Il Mulino.

BLAIS, A., y CARTY, R. K. (1987): "The impact of electoral formulae on the creation of majority governments», en Electoral Studies, 6: 209-218.

- (1991): «The psychological impact of electoral laws: measuring Duverger's elusive factor», en British Journal of Political Science, 21: 79-93.

CaCiagli, Mario, y CorbetTa, Piergiorgio (1987): «Introduzione», en M. Caciagli y P. Corbetta (eds.), Elezioni regionali e sistema politico nazionale. Italia, Spagna e la republica federale Tedesca, Bolonia: Il Mulino, pp. 9-23.

Duverger, Maurice (1974): Los partidos políticos, México: Fondo de Cultura Económica, 5. ${ }^{\mathrm{a}}$ reimp.

FERNÁNDEZ, Bernardo (1981): «El sistema electoral de las Comunidades Autónomas», en Siste$m a, 45: 75-88$.

Friedrich, Carl J. (1946): Constitutional Government and Democracy, Boston (trad. esp., Gobierno constitucional y democracia, Madrid: Instituto de Estudios Políticos, 2 vols.).

Gallagher, Michael (1991): "Proportionality, disproportionality and electoral systems», en Electoral Studies, 10: 33-51.

GroppI, Tania (1991): «Il sistemi elettorali a livello locale e autonomico in Spagna», en G. Rolla (ed.), Le forme di governo nei moderni ordinamenti policentrici. Tendenze e prospettive nell'esperienza costituzionale italiana espagnola, Milán: Giuffrè, pp. 279-323.

GUNTHER, Richard (1989): «Leyes electorales, sistemas de partidos y élites: el caso español», en REIS, 47: 73-106.

LaAkso, Markku, y TAagepera, Rein (1979): «Effective number of parties. A measure with application to west Europe», en Comparative Political Studies, 12: 3-27.

LIJPHART, Arend (1968): The politics of accomodation: pluralism and democracy in the Netherlands, Berkeley: Univ. of California Press.

- (1985): «The field of electoral systems research: a critical survey», en Electoral Studies, 4: 3-14.

- (1986): «Degrees of proportionality of proportional representation formulas», en B. Grofman y A. Lijphart (eds.), Electoral Laws and their political consequences, New York: Agathon Press, pp. 170-179.

- (1987): Las democracias contemporáneas. Un estudio comparativo, Barcelona: Ariel.

- (1990): «The political consequences of electoral laws, 1945-85», en American Political Science Review, 84: 481-496.

Montero, José R. (1992): «Las elecciones legislativas», en R. Cotarelo (ed.), Transición política y consolidación democrática en España (1975-1986), Madrid: CIS, pp. 243-297.

Montero, José R.; Llera, Francisco J., y TorCal, Mariano (1992): «Sistemas electorales en España: una recapitulación", en REIS, 58: 7-56.

Nohlen, Dieter (1981): Sistemas electorales del mundo, Madrid: Centro de Estudios Constitucionales.

- (1983): «Reforma del sistema electoral español: conveniencias, fórmulas y efectos políticos», en Revista de Estudios Politicos, 34: 61-68.

- (1991): Sistemas electorales y partidos politicos, multicopiado.

RAE, Douglas W. (1971): The political consequences of electoral laws, New Haven: Yale Univ. Press, 2. ${ }^{\mathrm{a}}$ ed.

Revenga, Miguel (1988): La formación de Gobierno en la Constitución Española de 1978, Madrid: Centro de Estudios Constitucionales.

SARTORI, Giovanni (1980): Partidos y sistemas de partidos, Madrid: Alianza.

- (1984): «La "leggi" sulla influenza dei sistema elettorali", en Rivista Italiana di Scienza Politica, 14: 3-40.

- (1986): «The Influence of electoral systems: faulty laws or faulty method?», en B. Grofman y A. Lijphart (eds.), Electoral laws and their consequences, op. cit., pp. 43-68. 
SHUGART, Matthew F. (1985): «The two effects of district magnitude: Venezuela as a crucial experiment», en European Journal of Political Research, 13: 353-364.

TAAGEPERA, Rein, y LAAKSO, Markku (1980): "Proportional profiles of west european electoral systems», en European Journal of Political Research, 8: 423-446.

TAagepera, Rein, y SHugart, Matthew F. (1990): Seats and votes. The effects and determinants of electoral systems, New Haven: Yale Univ. Press.

VALLÈs, Josep M. (1982): Reforma electoral i coordenades polítiques. Els condicionants de la normativa electoral a Espanya i a Catalunya, Barcelona: Fundació Jaume Bofill.

- (1987): "Quante Spagne elettorali? Dimensioni territoriali del fenomeno elettorale nella Spagna odierna", en M. Caciagli y P. Corbetta (eds.), Elezioni regionali..., op. cit., pp. 97127.

- (1988): «Los sistemas electorales subestatales en España: un balance (1979-1988)», ponencia presentada en el Seminario sobre Sistemi elettorali nelle regioni europee, Venecia (Italia).

- (1991): «Entre la regularidad y la indeterminación. Balance sobre el comportamiento electoral en España (1977-1990)», en J. Vidal-Beneyto (ed.), España a debate. La política, Madrid: Tecnos, pp. 27-43.

\begin{abstract}
With the basic features of the Spanish voting system having now been in place for 20 years and generalised at regional level for the last 15 , the system may be said to be institutionalised. In a politically decentralised state, any study of the way the voting system works must necessarily draw on its generic influence on the working of the political system and, more specifically, on electoral behaviour, representation, the party system and governability. This paper aims to contribute to explaining the terms of the debate on the Spanish voting system by providing a better understanding of the electoral effects and political consequences of the voting systems in Spain's 17 Autonomous Communities. More specifically, it tackles the following aspects: basic and differential features of the regional election systems, the sizes and coefficients of representation of the respective parliaments, the inequality and lack of proportion of the vote in relation to the size of the districts, minimum voting barriers and inclusion thresholds, a comparison of the main indicators of disproportion and, finally, the formats of the various regional party systems and the ensuing models of governability.
\end{abstract}

